\title{
Contraction of fibrin-derived matrices and its implications for in vitro human skin bioengineering
}

\author{
Andrés Montero $^{1}$ | Sonia Acosta ${ }^{1}$ | Rebeca Hernández ${ }^{2}$ | Carlos Elvira ${ }^{3}$ | \\ José Luis Jorcano $^{1,4}$ | Diego Velasco ${ }^{1}$ (i)
}

${ }^{1}$ Department of Bioengineering and Aerospace Engineering, Universidad Carlos III de Madrid (UC3M), Madrid, Spain

${ }^{2}$ Institute of Polymer Science and Technology, CSIC, Madrid, Spain

${ }^{3}$ Department of Applied Macromolecular Chemistry, Institute of Polymer Science and Technology, CSIC, Madrid, Spain

${ }^{4}$ Division of Epithelial Biomedicine, CIEMAT, Madrid, Spain

\section{Correspondence}

José Luis Jorcano and Diego Velasco, Department of Bioengineering and Aerospace Engineering, Universidad Carlos III de Madrid (UC3M), Madrid, Spain.

Email: jjorcano@ing.uc3m.es (J. L. J.) and

divelasc@ing.uc3m.es (D. V.)

Funding information

Cátedra Fundación Ramón Areces; Programa de Actividades de I + D Entre Grupos de Investigación de la Comunidad de Madrid, Grant/Award Number: S2018/BAA-4480; Programa Estatal de I + D + i Orientada a los Retos de la Sociedad, Grant/Award Numbers: MAT2013-42957-R, RTI2018-101627-B-I00; Proyectos de I + D Correspondientes al Programa Estatal de Fomento de la Investigación Científica y Ténica de Excelencia, Grant/Award Number: MAT2017-83014-C2-1-P

\begin{abstract}
It is well-known that fibroblasts play a fundamental role in the contraction of collagen and fibrin hydrogels when used in the production of in vitro bilayered skin substitutes. However, little is known about the contribution of other factors, such as the hydrogel matrix itself, on this contraction. In this work, we studied the contraction of plasma-derived fibrin hydrogels at different temperatures $\left(4,23\right.$, and $\left.37^{\circ} \mathrm{C}\right)$ in an isotonic buffer (phosphate-buffered saline). These types of hydrogels presented a contraction of approximately $30 \%$ during the first $24 \mathrm{hr}$, following a similar kinetics irrespectively of the temperature. This kinetics continued in a slowed down manner to reach a plateau value of $40 \%$ contraction after $10-15$ days. Contraction of commercial fibrinogen hydrogels was studied under similar conditions and the kinetics was completed after $8 \mathrm{hr}$, reaching values between 20 and 70\% depending on the temperature. We attribute these substantial differences to a modulatory effect on the contraction due to plasma proteins which are initially embedded in, and progressively released from, the plasma-based hydrogels. The elastic modulus of hydrogels measured at a constant frequency decreased with increasing temperature in 7-day gels. Rheological measurements showed the absence of a strain-hardening behavior in the plasma-derived fibrin hydrogels. Finally, plasma-derived fibrin hydrogels with and without human primary fibroblast and keratinocytes were prepared in transwell inserts and their height measured over time. Both cellular and acellular gels showed a height reduction of $30 \%$ during the first $24 \mathrm{hr}$ likely due to the above-mentioned intrinsic fibrin matrix contraction.
\end{abstract}

\section{KEYWORDS}

bilayered in vitro skin substitutes, contraction of fibrin hydrogels, fibrin hydrogel, plasmaderived fibrin hydrogel, skin tissue engineering

\section{1 | INTRODUCTION}

Over the last years, there has been a huge demand in the development and production of in vitro-engineered skin substitutes that mimic human skin, either to be used as grafts to restore the damaged skin or for the establishment of human-based in vitro skin models for toxicity, cosmetic, and pharmaceutical testing (Chua et al., 2016;
Groeber, Holeiter, Hampel, Hinderer, \& Schenke-Layland, 2011; MacNeil, 2007; Mathes, Ruffner, \& Graf-Hausner, 2014; Metcalfe \& Ferguson, 2007; Shevchenko, James, \& James, 2010; Shores, Gabriel, \& Gupta, 2007; Supp \& Boyce, 2005; Vig et al., 2017). As a result, acellular and cellular skin substitutes (containing keratinocytes, fibroblasts and adipocytes) as cellular monolayers (epidermal substitutes), cellular bilayers (dermoepidermal substitutes) and cellular trilayers 
(dermoepidermal-hypodermal substitutes) skin substitutes have been explored in this field (Auger, Lacroix, \& Germain, 2009; Stojic et al., 2019; Vig et al., 2017; Yu et al., 2019).

Cellular bilayered constructs containing dermal and epidermal components offer a promising approach to skin engineering since they allow the preparation of more sophisticated laboratory-grown skin substitutes which better resemble the structure and function of human skin (Caravaggi et al., 2003; Centanni et al., 2011; Curran \& Plosker, 2002; Llames et al., 2004; Llames et al., 2006; Meana et al., 1998; Stark et al., 2006; Still, Glat, Silverstein, Griswold, \& Mozingo, 2003). The production of bilayered artificial human skin usually involves the use of different types of scaffolds composed of natural (such as alginate, collagen, chitosan, fibrin and hyaluronic acid) and/or synthetic (such as poly(ethylene glycol), polycaprolactone, poly (vinyl alcohol), polylactic acid, and poly-L-lactic acid (Chaudhari et al., 2016; Jeong, Park, \& Lee, 2017; MacNeil, 2008; Norouzi, Boroujeni, Omidvarkordshouli, \& Soleimani, 2015; Priya, Jungvid, \& Kumar, 2008). Among them, collagen (the main component in the extracellular matrix [ECM]) and fibrin (derived from blood fibrinogen) hydrogels have been used extensively to provide structural and mechanical support (Ahmed, Dare, \& Hincke, 2008; Cen, Liu, Cui, Zhang, \& Cao, 2008; Glowacki \& Mizuno, 2008).

As an example of production of human artificial skin, our lab has engineered a human plasma-derived bilayered skin substitute containing primary human fibroblasts (hFBs) and keratinocytes (hKCs) from skin biopsies (Meana et al., 1998). These bilayered skin grafts have been successfully used to treat burns, as well as traumatic and surgical wounds in a large number of patients in Spain and for the generation of skin-humanized mouse models (Guerrero-Aspizua et al., 2010; Llames et al., 2004; Llames et al., 2006; MartinezSantamaria et al., 2013). Our lab has also developed a complete system (printer and bioinks) to 3D print this bilayered skin for clinical and commercial testing purposes (Cubo, Garcia, del Canizo, Velasco, \& Jorcano, 2016; Velasco, Quílez, Garcia, del Cañizo, \& Jorcano, 2018). In general, these skin substitutes formed by two layers, representing the dermis and the epidermis, were generated following the method developed in (Meana et al., 1998). The lower layer was a human plasma-derived fibrin hydrogel populated with hFBs and the upper layer was formed by $\mathrm{hKC}$, seeded confluent on the top of the plasma-derived fibrin hydrogel. At this point, for clinical use, the bilayered skin substitutes can be transplanted to the patients. After grafting, the layer of epidermal cells, now in contact with the air, can proliferate and differentiate to form all the layers of the epidermis, including the stratum corneum. A very similar process occurs when, for experimental purposes, human skin substitutes are grafted onto the back of immunodeficient mice (skin-humanized mice). For in vitro studies, the terminal differentiation of the epidermis is achieved by placing the culture in transwell inserts at the air-liquid surface for 17 days.

Although fibroblast-mediated matrix contraction is a parameter frequently used to examine fibroblast function, activity, and fate (Chen et al., 2005; Darby, Zakuan, Billet, \& Desmouliere, 2016; Ferrer-Mayorga et al., 2017; Javelaud, Laboureau, Gabison,
Verrecchia, \& Mauviel, 2003; Kopp et al., 2005; Li \& Wang, 2011; Tracy, Minasian, \& Caterson, 2016; Wynn \& Ramalingam, 2012; Yang et al., 2015), it constitutes a relevant limitation for the standardization of bilayered skin substitutes and their reliable application. This fibroblast-mediated contraction, in the case of skin cultures based on collagen and fibrin hydrogels, results in constructs that either lack attachment to the culture insert wall and/or suffer shrinking in the zaxis (Bell, Ivarsson, \& Merrill, 1979; Meana et al., 1998). Furthermore, skin substitutes present these phenomena even during manipulation, transport, or implantation, which can complicate their clinical use (Campbell, Bennett, Driscoll, \& Sawhney, 2005; Carriel et al., 2012; Haugh, Thorpe, Vinardell, Buckley, \& Kelly, 2012; Tuan, Song, Chang, Younai, \& Nimni, 1996). In addition, plasma-derived fibrin hydrogels low stiffness makes difficult their surgical handling (Haisch, Klaring, Groger, Gebert, \& Sittinger, 2002; Ruszymah, Chua, Mazlyzam, \& Aminuddin, 2011).

Several attempts such as physical modification, anchoring the hydrogels, caging in solid scaffolds, chemical modification or even introducing other synthetic or natural polymers, have been made to overcome the contractility of collagen and fibrin hydrogels in skin tissue engineering (Braziulis et al., 2012; Burmeister et al., 2016; Burmeister, Roy, Becerra, Natesan, \& Christy, 2018; Lotz et al., 2017; Mandrycky, Wang, Kim, \& Kim, 2016; Monaghan, Browne, SchenkeLayland, \& Pandit, 2014; Mori, Morimoto, \& Takeuchi, 2017; Natesan et al., 2019; Sriram et al., 2018; Stone, Wall, Natesan, \& Christy, 2018). In all these studies, it was considered that fibroblasts-mediated matrix contraction is the main component of this phenomenon (de Jesus \& Sander, 2014; Freyman, Yannas, Yokoo, \& Gibson, 2001; Grinnell, 2000; Jansen, Bacabac, Piechocka, \& Koenderink, 2013; Tuan et al., 1996; Zhu et al., 2001). However, the possibility that the collagen and fibrin matrices present an intrinsic, fibroblast-independent contraction has been poorly investigated (Zhang, Xu, Wei, Zhai, \& Li, 2013; Zhao et al., 2008).

Therefore, further characterization of the fibrin matrix is required to fully understand its behavior and discern the reason behind this excessive contraction, which hinders the usefulness of fibrin-based bilayered skin substitutes.

To this aim, we first studied the effect of different temperatures $\left(4^{\circ} \mathrm{C}\right.$ [storage temperature], $23^{\circ} \mathrm{C}$ [room temperature], and $37^{\circ} \mathrm{C}$ [tissue culture temperature]) on the contraction (mass loss), porosity, morphological structure, and mechanical properties of plasma-derived fibrin matrices free-floating in a commonly used isotonic buffer (phosphate-buffered saline [PBS]), using commercial fibrinogen hydrogels as controls. Then, to get closer to tissue culture conditions, the effect of the culture medium (Dulbecco's modified eagle medium [DMEM] supplemented with $10 \%$ fetal bovine serum [FBS] at $37^{\circ} \mathrm{C}$ ) on the contraction (mass loss) of free-floating plasma-derived fibrin hydrogels was analyzed.

Finally, the contraction of the bilayered skin substitutes was studied under their standard culture conditions, that is, within a transwell in which they remain attached to the walls and containing hFBs embedded in the dermal matrix (the plasma-derived fibrin hydrogel) and $h K C s$ on its surface. The results of all these experiments 
demonstrate that there is an intrinsic contraction due to the plasmaderived fibrin matrix itself, which under our experimental conditions is similar in magnitude to the contraction due to the cells, and that this effect has to be taken into account when using bilayered skin substitutes.

\section{2 | MATERIALS AND METHODS}

\section{1 | Materials}

Fresh frozen human platelet-poor plasma was provided by a local blood bank (Banco de Sangre del Centro Comunitario de Transfusión del Principado de Asturias [CCST], Spain) and were obtained according to the standards of the American Association of Blood Banks (Walker, 1993). For conservation purposes, plasma batches were aliquoted and stored at $-20^{\circ} \mathrm{C}$ (Brecher, 2005; Insenser, Martinez-Garcia, Nieto, San-Millan, \& Escobar-Morreale, 2010; Pinto et al., 2014). Commercial fibrinogen from human plasma was obtained from Sigma Aldrich and was stored at $-20^{\circ} \mathrm{C}$. Thrombin was obtained as a raw material from Sigma Aldrich, and was stored at $-20^{\circ} \mathrm{C}$ Each vial contained 10 National Institute of Health $(\mathrm{NIH})$ units of thrombin approximately. Calcium chloride $\left(\mathrm{CaCl}_{2}\right)$, sodium chloride $(\mathrm{NaCl}), \mathrm{PBS}$, and bovine serum albumin (BSA) were purchased from Sigma Aldrich. Amchafibrin (Rottafarm, Spain) was used as an antifibrinolytic agent. The Bio-Rad Protein Assay Dye Reagent Concentrate (Bio-Rad) was used for the Bradford assays.

\subsection{Preparation of plasma- and commercial fibrinogen-derived fibrin hydrogels}

Plasma-derived fibrin hydrogels were prepared following the protocol described by Meana et al. (1998). The experiments described in this article were performed using plasma from the same batch. Plasma aliquots of known fibrinogen concentration were thawed in a water bath at $37^{\circ} \mathrm{C}$. In a typical experiment, to prepare $0.813 \mathrm{ml}$ of plasma hydrogel in a glass vial, $400 \mu$ of plasma (with a fibrinogen concentration of $2.44 \mathrm{mg} / \mathrm{ml}$ ) $6.5 \mu \mathrm{l}$ of Amchafibrin, $341.5 \mu \mathrm{l}$ of saline ( $\mathrm{NaCl} 0.9 \%$ [wt/vol]), and $65 \mu \mathrm{l}$ of $\mathrm{CaCl}_{2}$ (prepared at $1 \% \mathrm{wt} /$ vol in saline) were sequentially added to the vial and mixed. The final concentrations of fibrinogen and $\mathrm{CaCl}_{2}$ in the plasma hydrogels were adjusted to 0.12 and $0.08 \%$ (wt/vol), respectively, by conveniently varying the plasma and saline volumes used. The solution was kept inside an incubator at $37^{\circ} \mathrm{C}$ and $5 \% \mathrm{CO}_{2}$ for $1 \mathrm{hr}$ to allow the gelation reaction to occur.

Commercial fibrinogen-derived fibrin hydrogels having the same final concentration of fibrinogen than plasma-derived hydrogels $(1.2 \mathrm{mg} / \mathrm{ml})$, were prepared as follows Commercial fibrinogen was dissolved in saline at $5.2 \mathrm{mg} / \mathrm{ml}$ by vortexing. To prepare the thrombin solution, $1 \mathrm{ml}$ of saline was added to each vial (containing $10 \mathrm{NIH}$ units of thrombin). The amount of thrombin needed to prepare the hydrogels was determined taking into account (Fischer, Ruf, \&
Mueller, 1995; Gaffney \& Edgell, 1995) that there are from 2 to $5 \mathrm{nmol}$ (of thrombin in human plasma, and that one $\mathrm{NIH}$ unit of thrombin is equivalent to $0.324 \pm 0.073 \mu \mathrm{g}$ of thrombin. In a typical experiment, to prepare a volume of $1.626 \mathrm{ml}$ of commercial fibrinogen hydrogel in a glass vial, the following components were added consecutively: $1063.25 \mu \mathrm{l}$ of saline, $130 \mu \mathrm{l}$ of $\mathrm{CaCl}_{2}$ [prepared at $1 \% \mathrm{wt} / \mathrm{vol}$ in saline]) (final concentration $0.08 \% \mathrm{wt} / \mathrm{wt}$ ), $44.75 \mu \mathrm{l}$ of thrombin prepared as described above, $13 \mu \mathrm{l}$ of Amchafibrin, and finally $375 \mu \mathrm{l}$ of previously prepared fibrinogen $(0.12 \% \mathrm{wt} / \mathrm{wt}$ final concentration). Once the fibrinogen was added and mixed with the other reagents, the vial was closed, and placed into an incubator at $37^{\circ} \mathrm{C}, 5 \% \mathrm{CO}_{2}$, and $40 \%$ relative humidity for 1 hour.

\subsection{Gelation time and kinetics}

Plasma- and commercial fibrinogen-derived fibrin hydrogel gelation times were determined first using the flip-flop method. This method is based on the free flowing of liquid inside the vials where hydrogels were prepared. The hydrogel mixtures were prepared and incubated as above described. Vials were then tilted every $30 \mathrm{~s}$. The time when there was no liquid left in the vial and the hydrogel remained stuck at the bottom was considered as the gelation time. Hydrogel gelation was also determined by turbidimetry and monitored using a SynergyTM HTX Multi-Mode Microplate Reader (Winooski, VT). (La First, to determine the most adequate wavelength to study the sol-gel transition. To this, absorbance was measured from 200 to $800 \mathrm{~nm}$, in $5 \mathrm{~nm}$ steps, at $37^{\circ} \mathrm{C}$. Two different samples were compared. (a) a homogenized solution of plasma, Amchafibrin, $\mathrm{NaCl}$, and $\mathrm{CaCl}_{2}$ with the concentrations described in Section 2.2, that will eventually reach the gel state and display an increase of absorbance, and (b) the same solution without $\mathrm{CaCl}_{2}$, which will not gelate. A similar procedure was followed for commercial fibrinogen hydrogels. Once the optimal wavelength was determined, in the microplate reader for $1 \mathrm{hr}$, at $37^{\circ} \mathrm{C}$, in $30 \mathrm{~s}$ intervals for the plasma hydrogels and $5 \mathrm{~s}$ intervals for the commercial fibrinogen hydrogels.

\subsection{Contraction of hydrogels in PBS at different temperatures}

The contraction of the hydrogels was assessed by mass loss. Plasma and commercial fibrinogen hydrogels were prepared in glass vials, detached by adding $2 \mathrm{ml}$ of PBS at $37^{\circ} \mathrm{C}$, placed into $60 \times 15 \mathrm{~mm}^{2}$ Petri dishes (Fisher Scientific) and submerged in a total volume of $7 \mathrm{ml}$ of PBS at different temperatures $\left(4,23\right.$, and $\left.37^{\circ} \mathrm{C}\right)$. At each time point, PBS was removed, the dishes carefully dried and the hydrogels weighted in a precision scale balance. Afterward, $7 \mathrm{ml}$ of fresh PBS were pipetted into the dish at the desired temperature and the dishes were incubated at the desired temperature until the next time point. Weight measurements were performed at 1, 2, 4, 6, 8, 24, 48, and $72 \mathrm{hr}$, and 5, 7, 10, 15, 21, and 30 days. The following equation was used to calculate the mass-swelling ratio: 


$$
\mathrm{SR} w=\frac{\mathrm{Mi}}{\mathrm{Mo}}
$$

where $\mathrm{SR}_{\mathrm{w}}$ is the mass swelling ratio, $\mathrm{Mi}$ is the mass of the hydrogel at each time point, and Mo is the mass of the hydrogel at time zero.

\section{5 | Degradation assay of fibrinogen hydrogels}

Commercial fibrinogen hydrogels degradation was assessed in PBS at $37^{\circ} \mathrm{C}$ by measuring the weight of the lyophilized hydrogels at different time points. Three hydrogels per time point were prepared as explained in Section 2.2 with a final volume of $1.5 \mathrm{ml}$ per gel. Samples were detached from the glass vials and incubated in $7 \mathrm{ml}$ of PBS at $37^{\circ} \mathrm{C}$ for $0,1,7,14$, and 21 days (in Petri dishes). At each time, hydrogels were removed from the incubator and freeze dried in a LABCONCO freeze dryer. Dried hydrogels were weighted twice in p35 dishes. Results were expressed as final dried weight in milligrams.

\subsection{Amount of cumulative protein released from hydrogels}

The Bio-Rad Protein Assay Dye Reagent Concentrate was used to determine the amount of cumulative proteins released from plasma and commercial fibrinogen hydrogels at the temperatures and time points used in the contraction studies (Section 2.4) described by Bradford (Bradford, 1976) and using BSA in PBS for the calibration curve (Zor \& Selinger, 1996).

To determine the amount of protein released from the hydrogels, media was removed from the corresponding Petri dish, and $5 \mu \mathrm{l}$ of collected media was mixed with $795 \mu \mathrm{l}$ of PBS and $200 \mu \mathrm{l}$ of Bradford reagent and the absorbance read in the spectrophotometer at $595 \mathrm{~nm}$. Solution was periodically collected for its analysis and then replaced by the same volume of fresh media for the rest of the time points.

\section{7 | Characterization of hydrogel structure}

Plasma and commercial fibrinogen hydrogels were structurally characterized through scanning electron microscopy (SEM). In order to maintain its internal morphology, samples were subjected to supercritical $\mathrm{CO}_{2}$ point drying reported elsewhere (Cohen, 1979; Niederman, Amrein, \& Hartwig, 1983; Syverud, Kirsebom, Hajizadeh, \& ChingaCarrasco, 2011). Plasma and commercial fibrinogen hydrogels at Time 0 were prepared and detached from the glass vials with distilled water $\left(\mathrm{dH}_{2} \mathrm{O}\right)$ at $37^{\circ} \mathrm{C}$ after $1 \mathrm{hr}$ of incubation at $37^{\circ} \mathrm{C}$ and $5 \% \mathrm{CO}_{2}$. Plasma hydrogels kept in PBS for $48 \mathrm{hr}$ at $4^{\circ} \mathrm{C}$ and for 7 days at $37^{\circ} \mathrm{C}$, were also analyzed. Hydrogels were dehydrated by sequential incubations in ethanol/water solutions according to the following procedure: hydrogels were first introduced in $20 \%$ ethanol for $2 \mathrm{hr}$. After this time, they were immersed in $40 \%$ ethanol for $2 \mathrm{hr}$ and then left overnight in $60 \%$ ethanol. Two more exchanges were made the next day: immersion in $80 \%$ ethanol for $2 \mathrm{hr}$ and then in $100 \%$ ethanol for the same time. Finally, hydrogels were stored overnight in $100 \%$ ethanol at $100 \% \mathrm{vol} / \mathrm{vol}$ and then transferred to p35 dishes.

Prior to characterization of the structure of the hydrogels by SEM, they subjected to $\mathrm{CO}_{2}$ supercritical drying by placing them into a supercritical reactor Thar R100W and gradually replacing ethanol with $\mathrm{CO}_{2}$. Subsequently, the temperature and the pressure of the system were raised during $10 \mathrm{~min}$ from $23^{\circ} \mathrm{C}$ and 60 bar to $35^{\circ} \mathrm{C}$ and 100 bars (supercritical point of $\mathrm{CO}_{2}$ is $31^{\circ} \mathrm{C}$ and $73.96 \mathrm{bar}$ ) and left for 90 min under $\mathrm{CO}_{2}$ supercritical conditions using a $5 \mathrm{~g} / \mathrm{min} \mathrm{CO}_{2}$ flow. Then, the system was slowly depressurized to room temperature and atmospheric pressure during $20 \mathrm{~min}$. Once dried, gels were immersed in liquid nitrogen to facilitate sectioning. Samples were fixed in the substrate and then sputter coating was performed. Samples were coated for $75 \mathrm{~s}$ with gold by using Leica EM ACE600 to minimize possible damage to the specimens and to improve topographical contrast for better SEM analysis. The dehydrated gels were imaged using a Philips XL30 SEM.

\subsection{Rheological measurements}

The rheological characterization of plasma-derived hydrogels was performed in a TA Instruments AR-G2 Rheometer (New Castle) using a sand-blasted aluminum plate ( $25 \mathrm{~mm}$ diameter). Disc-shaped gel samples were placed between the parallel plates and the gap between the plates was adjusted applying the minimum normal force to prevent slippage. A gap higher than $1,000 \mu \mathrm{m}$ was always reached after the sample relaxed until equilibrium. Measurements were performed on plasma-derived hydrogels immersed in PBS for 0,2 , and 7 days at 4 , 23 , and $37^{\circ} \mathrm{C}$, with the sample temperature being controlled and maintained using a Peltier device.

First, the storage modulus $\left(G^{\prime}\right)$ and the loss modulus $\left(G^{\prime \prime}\right)$ were measured as a function of strain by an oscillatory strain sweep with amplitudes ranging between 0.01 and $200 \%$ and at a frequency of $0.3 \mathrm{~Hz}$. These measurements were performed to determine the range of strain amplitudes over which the gels exhibited a linear region of viscoelasticity. A second measurement consisted of the dynamic frequency sweep between 0.01 and $10 \mathrm{~Hz}$ at a fixed strain, selected within the hydrogel linear region with the aim of obtaining the dependence of $G^{\prime}$ and $G^{\prime \prime}$ with frequency.

\section{9 | Comparison of the contraction of plasma- derived fibrin hydrogel in PBS and DMEM plus $10 \%$ FBS at $37^{\circ} \mathrm{C}$}

Mass swelling was used to study the contractile behavior of hydrogels at $37^{\circ} \mathrm{C}$ in PBS and in supplemented cell culture medium (DMEM/ $10 \%$ FBS). Plasma hydrogels were prepared in glass vials and incubated at $37^{\circ} \mathrm{C}$ for $1 \mathrm{hr}$ for gelation. Afterward, gels were removed from the vials as previously stated, poured into preweighted p35 
dishes, weighted in a precision balance, and returned to PBS or $\mathrm{DMEM} / 10 \% \mathrm{FBS}$ at $37^{\circ} \mathrm{C}$. Subsequent weight measurements were performed 2, 4, 6, 8, 24, 48, and $72 \mathrm{hr}, 5,7,10,15,21$, and 30 days. The mass swelling ratio was calculated using Equation (1) (Section 2.4).

\subsection{0 | Primary hKCs and hFBs culture}

hFBs and hKCs obtained from skin biopsies of healthy donors were obtained from the collections of biological samples of human origin registered in the "Registro Nacional de Biobancos para Investigación Biomédica del Instituto de Salud Carlos III." In this work, we have used cells from a single donor. hKCs were cultured following previously described methods (Rheinwatd \& Green, 1975) as modified by our laboratory (del Rio et al., 2002; Meana et al., 1998). The growing medium for hKCs was a 3:1 mixture of DMEM (GIBCO-BRL) and HAM'S F12 (GIBCO-BRL) (hKC medium) containing 10\% of FBS, $0.1 \mathrm{nM}$ choleric toxin, $2 \mathrm{nM}$ T3, $5 \mathrm{mg} \mathrm{ml}^{-1}$ insulin, $0.4 \mathrm{mg} \mathrm{ml}^{-1}$ hydrocortisone, and $10 \mathrm{ng} \mathrm{ml}^{-1}$ EGF (Sigma, St Louis, MO) and Primocyn $1 \%$ antibiotic. hFBs were cultured in DMEM (BiochromKG) containing $10 \% \mathrm{FBS}$ and $1 \%$ penicillin/streptomycin.

\subsection{1 | Contraction of plasma-derived fibrin hydrogels in the z-axis under in vitro skin culture conditions}

Plasma hydrogels were prepared inside transwells in order to generate dermoepidermal skin constructs in vitro following the protocol previously reported by Meana et al. (1998). Human primary fibroblasts were incorporated at a 20,000 cells/ml density and hydrogels were left incubating for $1 \mathrm{hr}$. Human primary keratinocytes were later seeded after $24 \mathrm{hr}$ on top of the plasma hydrogels at a density of 1.5 million cells in $1 \mathrm{ml}$. Keratinocytes were left to sink and attach to the hydrogels surface for $48 \mathrm{hr}$ before transferring the whole system into deep-well plates. Medium was then removed from the top of transwells in order for the keratinocyte differentiation to start in contact with the air. This air liquid interface served as the system to generate organotypic skin constructs.

For comparison, we studied the contraction of plasma hydrogels without cells under similar conditions. Photographs were taken of the profile of the transwells in the presence of a ruler that acted as scale for the measurement of the height of the hydrogels during the differentiation process. Defined time points were $0 \mathrm{hr}, 4 \mathrm{hr}, 6 \mathrm{hr}, 8 \mathrm{hr}$, $24 \mathrm{hr}, 48 \mathrm{hr}, 72 \mathrm{hr}, 7$ days, 10 days, 15 days, and 21 days. Images were analyzed with the ImageJ software.

\subsection{2 | Statistical analysis}

Experiments were performed in triplicates unless otherwise specified in the figure legends. Data are expressed as means $\pm S D$. Statistical analysis was performed using the IBM SPPS software. Student's $t$ test (Student, 1908) was used to determine the statistical significance of result differences. Three levels of significance were assessed: ${ }^{*} p<.05$; ${ }^{* *} p<.01 ;{ }^{* * *} p<.001$.

\section{3 | RESULTS}

\subsection{Gelation kinetics}

Hydrogel gelation time was studied using the flip-flop method. The results show a significant difference between the gelation time of plasma hydrogels (13.4 $\pm 2.4 \mathrm{~min}$ ) and commercial fibrinogen hydrogels (3.4 $\pm 0.5 \mathrm{~min}$ ) (Figure 1a). Characterization of the gelation process was further assessed through the study of the polymerization kinetics by UV spectroscopy. The change in optical density (OD) for both hydrogel types was dynamically measured every $30 \mathrm{~s}$ to accurately determine the kinetics of the polymerization reaction. This is a common technique used by other groups that have followed the polymerization of fibrin hydrogels obtained from both commercial fibrinogen (Wedgwood, Freemont, \& Tirelli, 2013; Weigandt et al., 2012) and fibrinogen isolated from human plasma (Zhao et al., 2008). In order to determine the most adequate wavelength to follow this process, we performed UV scans from 200 to $800 \mathrm{~nm}$ before and after gelation.

In the case of commercial fibrinogen, the absorbance difference between solution and hydrogel was of $1 \mathrm{OD}$ at $350 \mathrm{~nm}$ (Figure 1b) whereas in the case of plasma hydrogels, this difference was of 0.5 $\mathrm{OD}$ at $325 \mathrm{~nm}$ (Figures $1 \mathrm{~b}$ and S1). These two wavelengths were then selected to follow the gelation experiments at $37^{\circ} \mathrm{C}$. These wavelengths fall inside the ones previously reported in the literature that range between 300 and $550 \mathrm{~nm}$, with lower wavelength being unsuitable due to potential interference with proteins and DNA (Turner, McLennan, Bates, \& White, 2007). To study the gelation kinetics, the components for both commercial fibrinogen and plasma hydrogels were mixed and quickly transferred into the plate reader. The commercial fibrinogen hydrogels displayed a quick increase of absorbance, reaching a plateau at around $1 \mathrm{AU}$ after approximately $11 \mathrm{~min}$ (Figure 1b). The results for plasma hydrogels were quite different, showing a long initial lag phase, in which no change in absorbance was recorded, followed by a polymerization phase reaching a plateau at around 0.5 AU after approximately $25 \mathrm{~min}$ (Figure $1 \mathrm{~b}$ ).

\subsection{Contraction and protein release studies at different temperatures}

The results corresponding to the contraction studies performed on plasma-derived and commercial fibrinogen hydrogels are depicted in Figure 2a,b, respectively. The results obtained for both types of hydrogels showed that matrices contract after incubation in an aqueous environment at the studied temperatures $\left(4,23\right.$, and $\left.37^{\circ} \mathrm{C}\right)$. Plasma-derived hydrogels shrank following a similar behavior, 
irrespectively of the temperature (Figure 2a). They presented a relatively rapid contraction of approximately $25 \%$ during the first $8 \mathrm{hr}$ followed by a slower step in which they contract until reaching a plateau of approximately $35 \%$ after 10 days.

Fibrinogen hydrogels, used as controls and exposed to the same temperatures, also presented a deswelling behavior albeit clearly different from that shown by plasma-derived gels (Figure $2 b$ ). On the one hand, they only displayed the more pronounced, first $8 \mathrm{hr}$ step, On the other hand, they reached quite different contraction values depending on the temperature, being most dramatic the $70 \%$ mass loss found at $4^{\circ} \mathrm{C}$. Surprisingly, we did not find a direct correlation between contraction and temperature.

To find out whether the mass loss displayed by commercial fibrinogen hydrogels was, at least in part, due to degradation, we measured the weight of hydrogels incubated for different times (1, 7, 14, and 21 days) in $\mathrm{PBS}$ at $37^{\circ} \mathrm{C}$ and thereafter lyophilized. Results show that these hydrogels do not lose any statistically significant weight even after 21 days under these conditions (Figure 2c). The initial weight of the hydrogels was slightly below $15 \mathrm{mg}$ and was maintained throughout the experiments with minor variations.

Bradford assays were performed to obtain a deeper insight into the deswelling behavior of the plasma-derived hydrogels. A complication when studying plasma-derived hydrogels is that plasma contains a complex mixture of proteins among which albumin, globulins, and fibrinogen are the most abundant. While fibrinogen gives rise to the fibrin matrix, the rest of proteins are entrapped inside the hydrogel and are expected to be release from it into the surrounding PBS buffer along the time.
The results showed similarly shaped curves of protein release at the three investigated temperatures $\left(4,23\right.$, and $37^{\circ} \mathrm{C}$ ) (Figure $2 \mathrm{~d}$ ). The three curves reached a plateau at approximately $48 \mathrm{hr}$ although, as could be expected, both the amount of released protein and the initial slope of the curves increased with increasing temperature. In agreement with the data shown in Figure $2 \mathrm{~d}$, commercial fibrinogen hydrogels failed to show any protein release. Knowing the initial volume of human plasma used to prepare the plasma-derived hydrogels and the published concentration of total protein present in plasma (Arnaud, 2004; Buis et al., 1996), we estimate that our gels contain initially $25 \mathrm{mg}$ of proteins. Therefore, we were able to determine the percentage of plasma proteins released from the hydrogels incubated at the different temperatures $\left(4^{\circ} \mathrm{C}: 15 \mathrm{mg}, 60 \% ; 23^{\circ} \mathrm{C}\right.$ : $20 \mathrm{mg}, 80 \%$; and $37^{\circ} \mathrm{C}: 22.5 \mathrm{mg}, 90 \%$ ) after 30 days. As Figure $2 \mathrm{~d}$ shows, commercial fibrinogen hydrogels do not present any protein release detectable by this assay.

Statistically significant differences were found at $48 \mathrm{hr}$ (the plateau of the curves) when comparing the protein release values at 4 and $23^{\circ} \mathrm{C}(p<.001)$ and when comparing at the same time point the values at 4,23 , and $37^{\circ} \mathrm{C}(p<.01$. In parallel experiments to those described, the evolution of plasma-derived hydrogels at $4^{\circ} \mathrm{C}$ was visually observed and photographed at different time points (Figure 2e). They presented a progressive decrease in size and a change in color, from an initial yellow color to a final pale, semitransparent one. These changes are coherent with the contraction and protein release data of Figure 2a,d, respectively. Furthermore, polyacrylamide gel electrophoresis corroborated the Bradford assay results (Figure S2).
FIGURE 1 (a) Gelation time at $37^{\circ} \mathrm{C}$ for commercial fibrinogen (left) and plasma hydrogels (right) using the flip-flop method. (b) Gelation kinetics for commercial fibrinogen and plasma hydrogels using UV spectrometry at 325 and $350 \mathrm{~nm}$, respectively. Data expressed as mean $\pm S D, n=8$ (a)

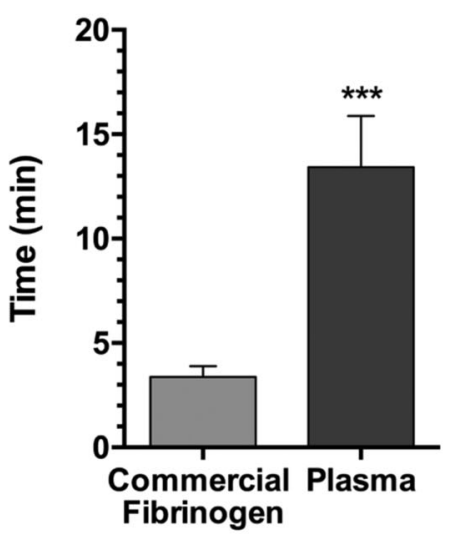

(b)
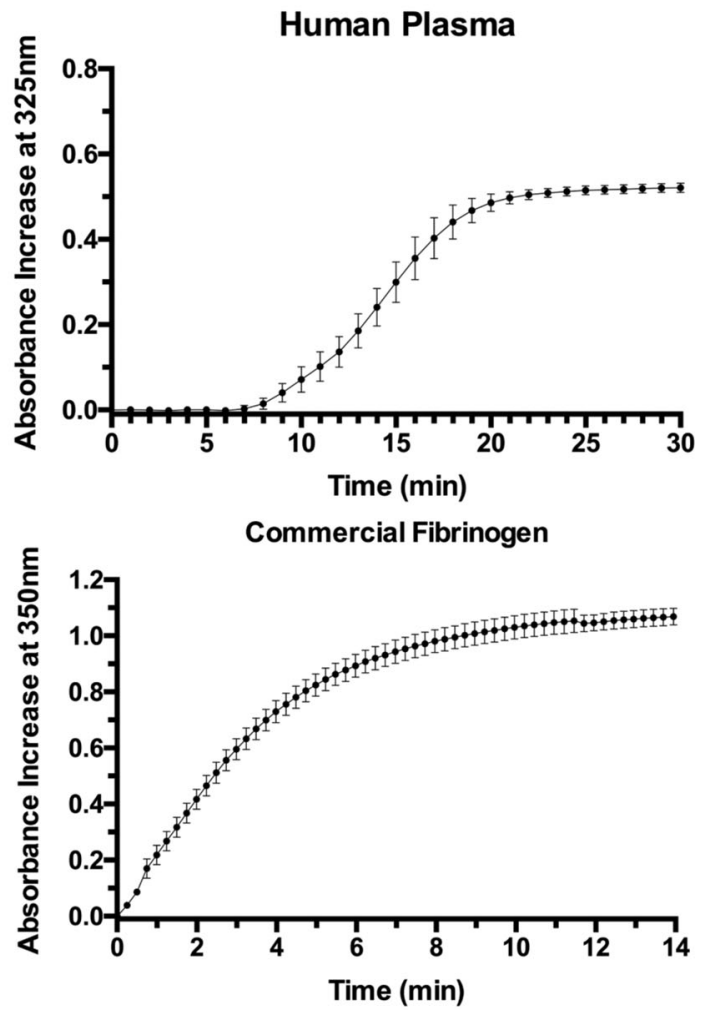
(a)

\section{Plasma Contraction}

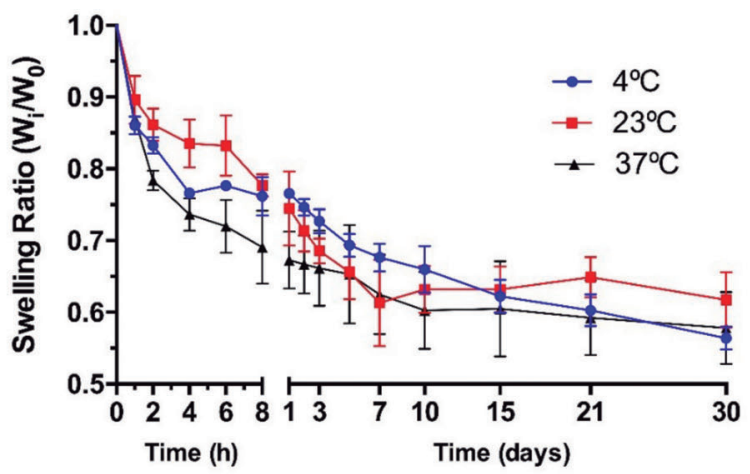

(c)

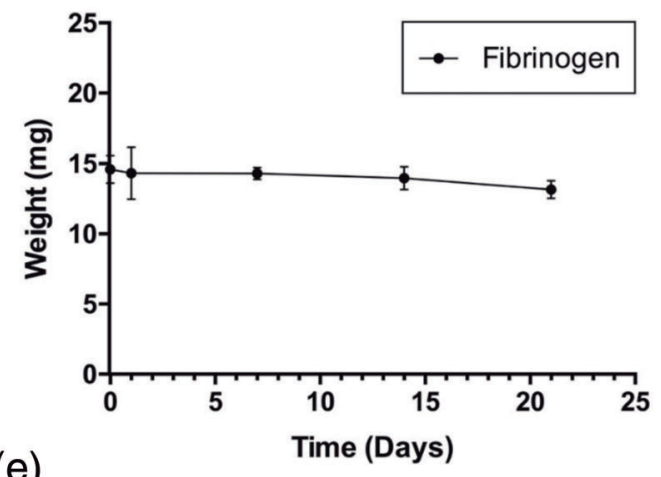

(e)

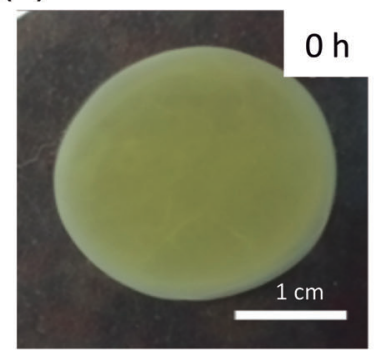

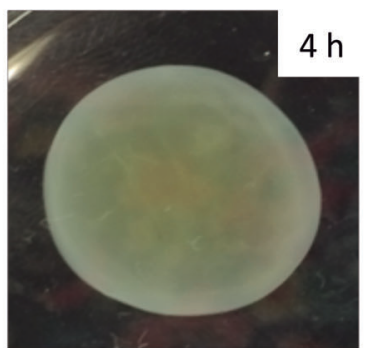

(b)

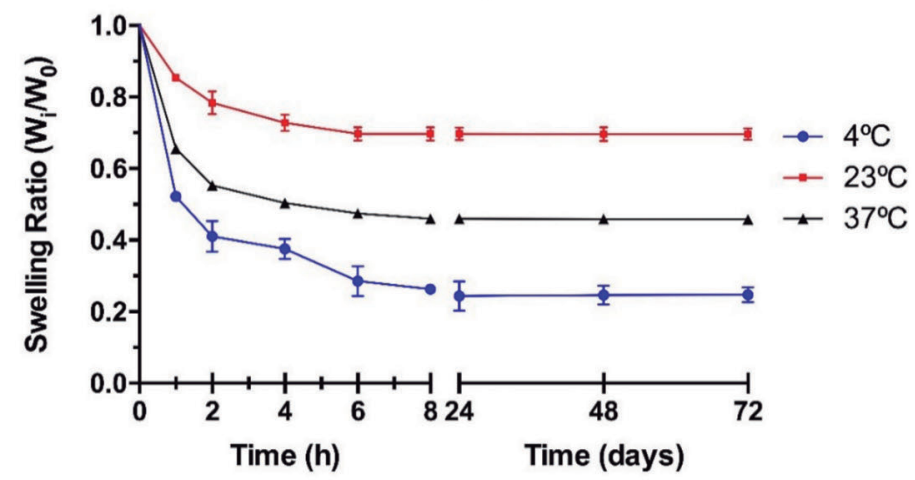

(d)

\section{Commercial Fibrinogen Contraction}

Protein release - Bradford Assay
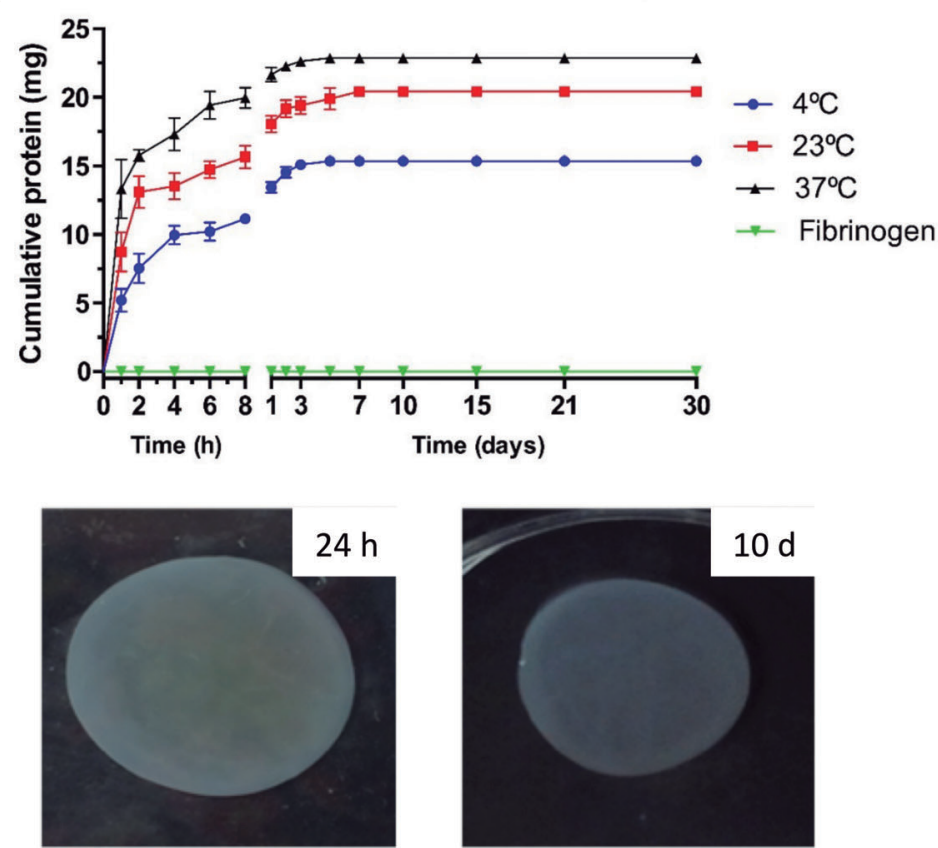

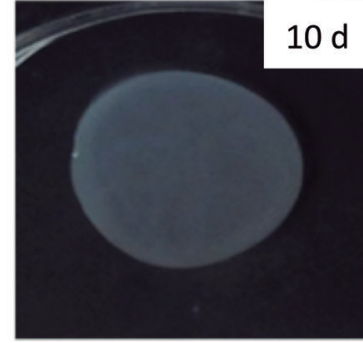

FIGURE 2 (a) Plasma hydrogels contraction, measured as mass loss, in PBS at 4, 23, and $37^{\circ} \mathrm{C}$. (b) Commercial fibrinogen hydrogels contraction, measured as mass loss, in PBS at 4,23 , and $37^{\circ} \mathrm{C}$. The plateau values at $48 \mathrm{hr}$ are statistically significantly different ( $p<.01$ ). (c) Commercial fibrinogen hydrogel degradation assay for 21 days in phosphate-buffered saline (PBS) at $37^{\circ} \mathrm{C}$. (d) Cumulative protein release for hydrogels incubated at 4,23 , and $37^{\circ} \mathrm{C}$ as measured by the Bradford assay. (e) Representative plasma-derived hydrogels incubated at $4^{\circ} \mathrm{C}$ in PBS for different times: $0 \mathrm{hr}, 4 \mathrm{hr}, 24 \mathrm{hr}$, and 10 days, showing the shrinkage, loss of color, and increased transparency that they experiment. Data are expressed as mean $\pm S D, n=4$. Scale bar $=1 \mathrm{~cm}$

\section{3 | Morphological characterization by SEM}

Comparison of Figure 3a,d demonstrates clear structural differences between untreated plasma-derived and commercial fibrinogen hydrogels. Whereas the later display smooth, tangled, and clearly delineated fibers with a diameter of $94 \pm 19.08 \mathrm{~nm}$ (Figure S4), the former's fibers are stiffer, irregular, and thicker $(280 \pm 47.53 \mathrm{~nm}$ in diameter). No differences in fiber thickness nor porous structure was found between the plasma hydrogels after 7 days at $37^{\circ} \mathrm{C}$ and the initial commercial fibrinogen hydrogels (Figure $3 \mathrm{c}, \mathrm{d}$ ). Finally, plasma-derived hydrogels treated for $48 \mathrm{hr}$ at $4^{\circ} \mathrm{C}$ present an intricate structure, somewhat reminiscent of that of untreated samples but more irregular, and with less defined fibers (Figure 2b).

\subsection{Rheological characterization}

Rheological experiments were designed to understand the performance of the hydrogels at different temperatures $\left(4,23\right.$, and $\left.37^{\circ} \mathrm{C}\right)$ in terms of shear forces and Representative results corresponding to strain sweep tests were carried out on untreated plasma-derived hydrogels (control gels, $0 \mathrm{hr}$ ), and plasma-derived hydrogels maintained at different temperatures during $48 \mathrm{hr}$ (Figure 4a). As a 

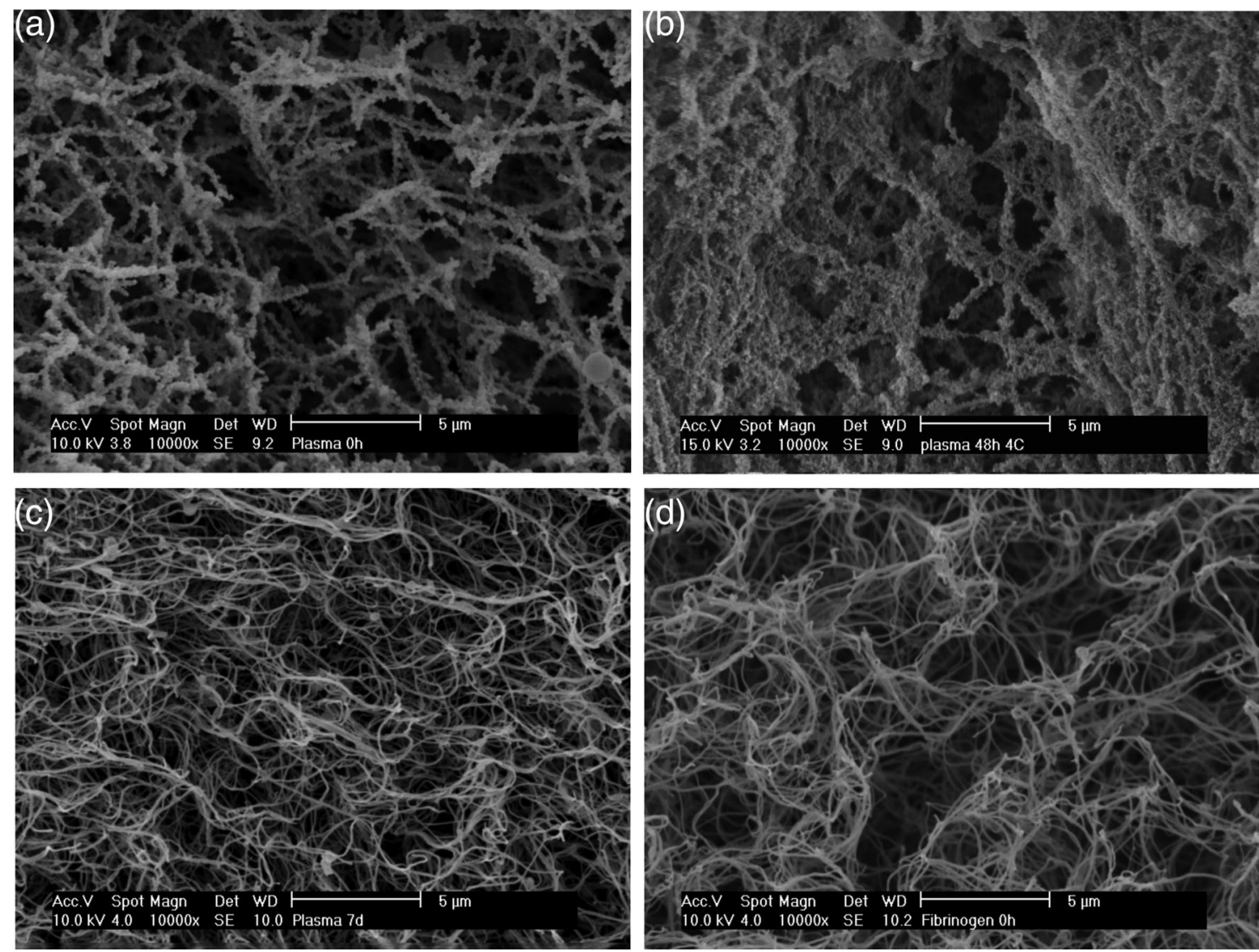

FIGURE 3 Scanning electron microscope (SEM) images of untreated plasma hydrogels (at $0 \mathrm{hr}$ ) (a); plasma-derived hydrogels treated for 48 hr in phosphate-buffered saline (PBS) at $4^{\circ} \mathrm{C}$ (b); plasma-derived hydrogels treated for 7 days in $\mathrm{PBS}$ at $37^{\circ} \mathrm{C}$ (c); and untreated commercial fibrinogen hydrogels (at $\mathrm{O} \mathrm{hr}$ ) (d)

general behavior, all samples present $G^{\prime}$ (elastic modulus) $>G^{\prime \prime}$ (viscous modulus) up to a critical deformation, $\gamma_{0}$, estimated as the point where the slope of the stress-strain curves change dramatically and $G^{\prime}$ and $G^{\prime \prime}$ rapidly decrease with increasing strain, causing the nonlinearity $\gamma_{0}$ represents the minimum strain needed to induce flow, and the crossover point between $G^{\prime}$ and $G^{\prime \prime}$ indicates the change from a solid-like to a liquid like behavior. Focusing on the evolution of $G^{\prime}$ with strain, none of the samples present strain-hardening behavior, that is an increase of $G^{\prime}$ with strain.

Focusing on the evolution of $G^{\prime \prime}$ with strain, it is observed that all the samples present a constant value of $G^{\prime \prime}$ up to a value of strain here $G^{\prime \prime}$ starts to increase giving rise to a maximum, followed again by a decrease (Figure 4a) A representation of $G^{\prime \prime}$ as a function of temperature obtained for plasma-derived hydrogels after $48 \mathrm{hr}$ and 7 days is shown in Figure 4b. Regardless the treatment time ( $48 \mathrm{hr}$ or 7 days) $G^{\prime \prime}{ }_{\text {max }}$ decreases with temperature being the values obtained for $G^{\prime \prime}$ max for gels after $48 \mathrm{hr}$ higher than the values obtained for $G^{\prime \prime}$ for gels after 7 days of treatment. In order to get further insight on the effect of temperature and time of incubation on the elastic properties of the hydrogels, $G^{\prime}$ and $G^{\prime \prime}$ were measured as a function of frequency. The results corresponding to hydrogels maintained at different temperatures for $48 \mathrm{hr}$ are depicted in Figure 4c. All the samples present gellike behavior which is characterized by (a) values of the elastic modulus higher than the corresponding to the viscous modulus $\left(G^{\prime}>G^{\prime \prime}\right)$ in the frequency range studied and (b) $G^{\prime}$ and $G^{\prime \prime}$ are independent of frequency in the studied frequency range.

The values of the moduli measured at a frequency of $0.3 \mathrm{~Hz}$ were analyzed for s plasma-derived hydrogels incubated at 4,23 , and $37^{\circ} \mathrm{C}$ during $48 \mathrm{hr}$. As Figure 4c shows, these moduli do not change appreciably with temperature. In contrast, the elastic moduli measured after 7 days of treatment show significantly different results. For all studied temperatures, the elastic moduli decrease with respect to the control gel $(0 \mathrm{hr})$ except for the gel maintained at $4{ }^{\circ} \mathrm{C}$ for which the elastic moduli remain unaffected by the thermal treatment (Figure $4 d$ ).

\subsection{Comparison of the contraction of plasma- derived fibrin hydrogels in PBS and DMEM plus10\% FBS at $37^{\circ} \mathrm{C}$}

To analyze their swelling behavior under situations similar to those found in tissue culture, plasma-derived hydrogels were incubated at $37^{\circ} \mathrm{C}$ in PBS (as a control) and in supplemented culture medium (DMEM plus $10 \%$ FBS) for 30 days. It was found (Figure $5 a$ ) that, as expected, PBS control hydrogels showed a deswelling (measured as mass loss) kinetics very similar to that already described under similar conditions (see Figure 2a), characterized by a rapid 
(a)

Strain Sweep 48h

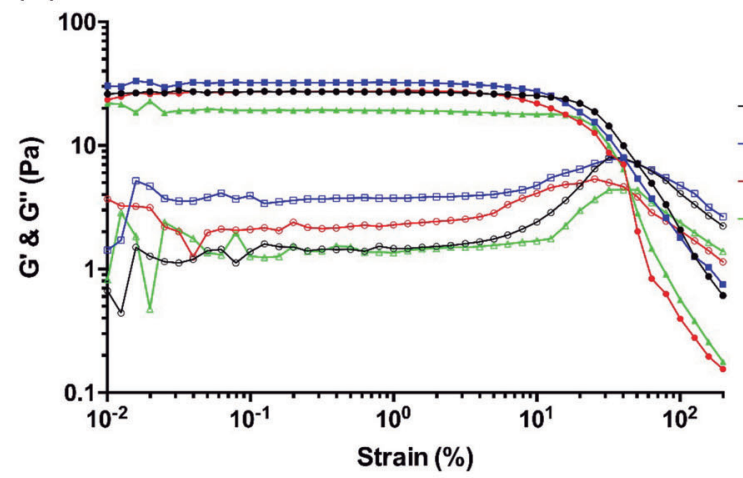

(c)

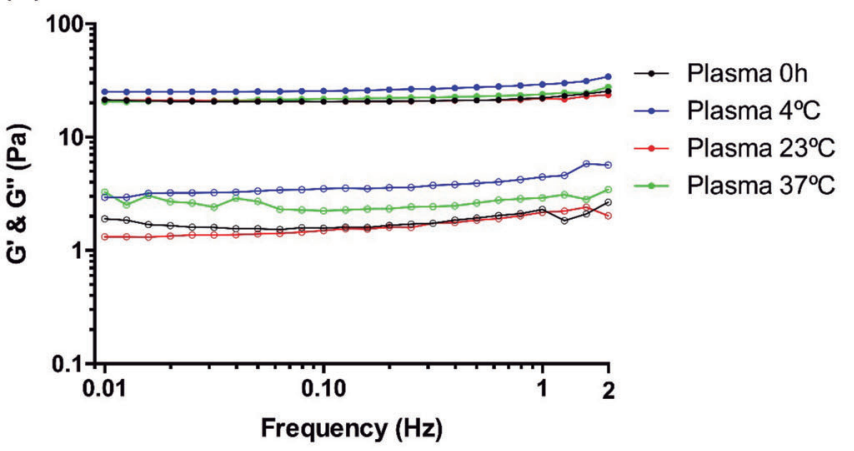

(b)

G" maximum

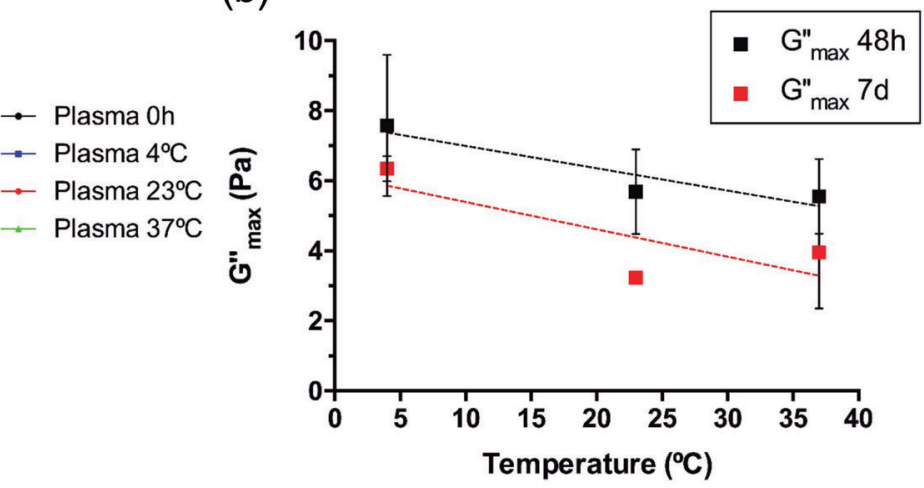

(d)

Elastic Modulus

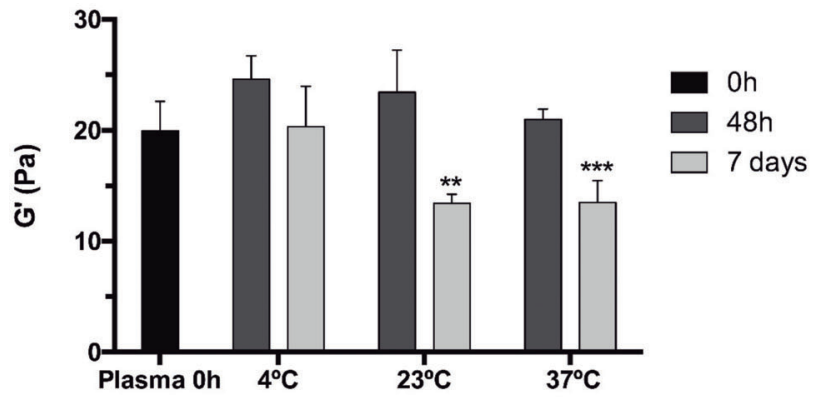

FIGURE 4 (a) Strain sweep test of plasma-derived hydrogels at different temperatures $\left(4,23\right.$, and $\left.37^{\circ} \mathrm{C}\right)$ after $48 \mathrm{hr}$ of incubation and control (plasma hydrogels at $0 \mathrm{hr}$ ). $G^{\prime}$ closed symbols, $G^{\prime \prime}$ open symbols. (b) Representation of $G^{\prime \prime}$ max obtained from strain sweep tests of plasma-derived fibrin gels incubated at different temperatures for $48 \mathrm{hr}(-)$ and 7 days ( $)$. Dashed lines are a guide for the eye. (c) Frequency sweep test. Samples are as in (a). Elastic moduli for hydrogels maintained at different temperatures $\left(4,23\right.$, and $\left.37^{\circ} \mathrm{C}\right)$ for $48 \mathrm{hr}$ and 7 days. For comparison, the elastic modulus corresponding to plasma-derived hydrogels at $0 \mathrm{hr}$ of incubation is also shown. Data expressed as mean $\pm S D, n=3$

(a)

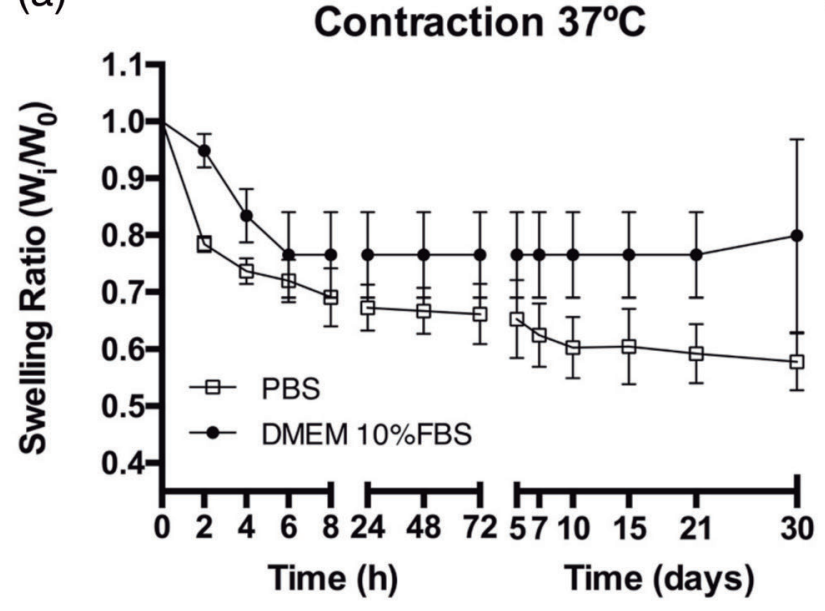

first phase of contraction (approximately 30\% mass loss) during the first 8-24 hr followed by a slow contraction phase (approximately $40 \%$ mass loss after 30 days). However, hydrogels incubated in supplemented culture medium only suffered the first, rapid contraction step. The two deswelling curves presented statistically significant differences, at least at the longer times (for instance, $p<.05$ at 21 days). (b)
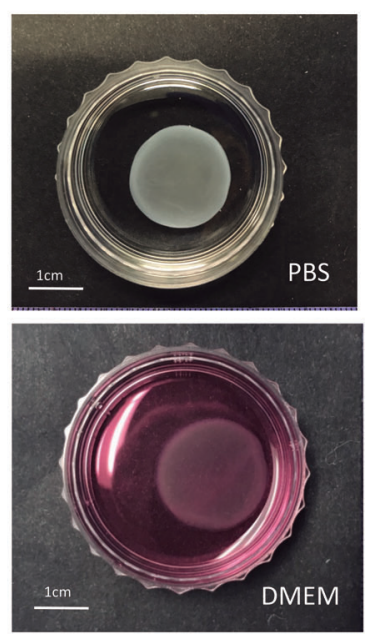

FIGURE 5 (a) Hydrogel contraction measured as mass loss comparing plasma-derived hydrogels incubated in phosphate-buffered saline (PBS) or in supplemented cell culture medium (Dulbecco's modified eagle medium (DMEM] supplemented with $10 \%$ fetal bovine serum [FBS]) at $37^{\circ} \mathrm{C}$. (b) Plasmaderived fibrin hydrogels free-floating in PBS and DMEM plus 10\% FBS at $24 \mathrm{hr}$ where the changes in weight were measured. Data expressed as mean $\pm S D$. $n=3$

\subsection{Contraction of plasma-derived fibrin hydrogels in the z-axis under in vitro skin culture conditions}

Until now, in this article, we have analyzed the behavior of acellular, free-floating hydrogels, either in glass vials or in Petri dishes. Now we want to study them under fully in vitro skin culture conditions, that is, 
FIGURE 6 (a) Hydrogels contraction in the z-axis in the presence and absence of human fibroblasts (hFBs) and keratinocytes (hKCs), incubated in Dulbecco's modified eagle medium (DMEM) supplemented with $10 \%$ fetal bovine serum (FBS) during 21 days. (b) Photograph of the culture system. (c) Scheme showing the different phases of the in vitro generation of a skin substitutes (plasma gels with hFB and hKC): (1) Human plasmaderived fibrin hydrogels containing embedded $\mathrm{hFBs}$ are generated in transwell inserts. (2) After $24 \mathrm{hr}$, hKCs are seeded at confluency on top of the hydrogels. (3) After additional $48 \mathrm{hr}$, the culture is placed at the air-liquid interface. (4) Full epidermal differentiation is achieved after 17 days of culture at the liquid-air interface. Data in (a) are expressed as mean $\pm S D . n=3$ (a)

Contraction in the Z-axis

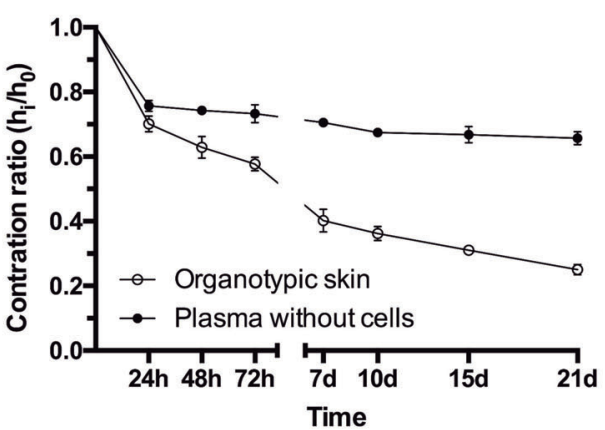

(b)

(c)

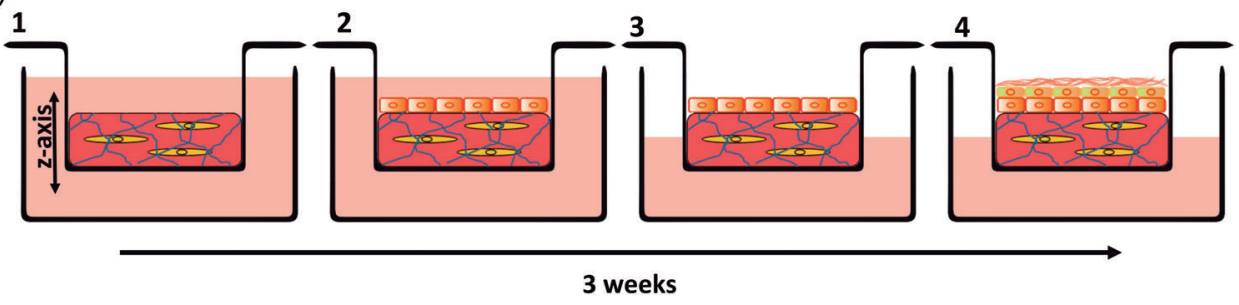

at $37^{\circ} \mathrm{C}$, in supplemented DMEM cell culture medium, containing cells and inside transwell inserts. Under these conditions, fibrin-derived hydrogels do not detach from the transwell walls and contraction is manifested as a decrease in gel height. To this, plasma-derived hydrogels, with and without skin cells, were prepared in transwell inserts following the standard in vitro skin equivalent generation protocol described in Section 2.11, and their height was measured using ImageJ software. Interestingly, the z-axis hydrogel contraction during the initial $24 \mathrm{hr}$ was very similar regardless of the presence of hFBS (Figure 6a). After the initial $24 \mathrm{hr}$, the effect of the cells contracting the matrix becomes apparent, making the hydrogels containing hFBs and hKCs to contract up to $70 \%$ after 21 days.

\section{4 | DISCUSSION}

In order to improve the usefulness of fibrin-based hydrogels and try to find ways to overcome some of their current drawbacks, in particular the contraction they experiment with time, in this work we have analyzed relevant processes that take place during their in vitro production and experimental and clinical use. We are particularly interested in fibrin hydrogels generated from human blood plasma because they contain a complex mixture of proteins and lipids entrapped in the fibrin matrix, which are known to be relevant for wound healing and, in particular, for cell proliferation and migration. Given that this complexity greatly complicates their analysis, we have used commercial fibrinogen-based hydrogels as controls to try to discern the cause of the observed results.

Regarding gelation, the two types of hydrogels behaved quite differently. For instance, commercial fibrinogen gels polymerized quicker and were quite fragile likely due, at least in part, to its very low fibrinogen concentration $(1.2 \mathrm{mg} / \mathrm{ml}$, to match the fibrinogen concentration used in the plasma-derived hydrogels) as compared to the concentrations usually reported in the literature $(10-20 \mathrm{mg} / \mathrm{ml})$. On the contrary, the proteins and lipids trapped in plasma-derived hydrogels provide them with mechanical stability and induce delayed gelation time probably due to steric effects (Arnaud, 2004).

To determine gelation time and kinetics, two frequently used methods, flip-flop and optical absorbance (turbidimetry), were followed. The first one is very quick and easy to perform but it gives information about whether the gels had formed just based on visual inspection. Even so, it indicated that commercial fibrinogen gels polymerize in a much shorter time (about $3.5 \mathrm{~min}$ ) than plasma-based gels (about $13.5 \mathrm{~min}$ ) (Figure 1a). These times are manifestly shorter than those measured by turbidimetric assays (Figure 1b), indicating that polymerization was far from completed. This last technique, based on light absorbance related to fibril formation, fibril structure, and fibrilfibril interactions (Kreger et al., 2010) provides quantified parameters such as kinetics shape, lag time, half-time, and final absorbance. As seen in Figure 1b, according to this technique, the two types of hydrogels follow very different polymerization kinetics, both qualitatively (e.g., lag time and shape of polymerization curves) and quantitatively (e.g., 
polymerization rate and final absorbance). Besides potential differences in fibrinogen characteristics related to the different sources of the material, we attribute these differences to two causes as follows:

1 The already mentioned proteins and lipids entrapped in plasmaderived hydrogels could be responsible of the larger polymerization times and lower OD observed in plasma-based gels by steric effects (Arnaud, 2004) and the color/turbidity they confer to human plasma (as an indication, see 0 time in Figure 2e), respectively.

2 Although we use physiological amounts of thrombin to induce the polymerization of commercial fibrinogen gels this thrombin is readily active while the complete coagulation cascade has to be triggered in the case of plasma hydrogels upon calcium addition.

Given that contraction is possibly the main drawback of plasmaderived fibrin hydrogels, we devoted a good deal of our effort to characterize this phenomenon. Unexpectedly, these gels contracted in an aqueous medium (PBS) with a similar temporal kinetics over a range of temperatures $\left(4,23\right.$, and $\left.37^{\circ} \mathrm{C}\right)$ of experimental and clinical relevance. They showed a bi-phasic deswelling behavior with an initial quick $25 \%$ loss of mass during the first $24 \mathrm{hr}$ followed by a slower step in which they reach a plateau of approximately $35 \%$ mass loss after 10 days (Figure 2a).

Control commercial fibrinogen hydrogels behaved in a remarkably different manner. Although they contracted at the studied temperatures, they presented only the first $24 \mathrm{hr}$ deswelling step and they reached quite different contraction values depending on the temperature (Figure 2b). Regarding this contraction, Zhao et al. (2008) have reported a weight loss of $80 \%$ after 3 days of incubation in PBS at $37^{\circ} \mathrm{C}$ for commercial fibrinogen hydrogels at different concentrations. The authors attributed this behavior to fibrinogen dissolution due to the ionic strength of PBS. However, we do not detect such dissolution of the fibrinogen hydrogels with time since we do not observe any protein release in the Bradford assays (Figure 2d) nor weight loss when they are lyophilized and weighted at different time points (Figure 2c). Altogether, these data demonstrate that commercial fibrinogen hydrogels, at least in our conditions, experience a temperaturesensitive intrinsic contraction during about $24 \mathrm{hr}$ and suggest that there must be additional reasons that explain the behavior of plasmaderived gels.

One of these reasons could be a modulatory effect exerted by the proteins and lipids embedded in plasma-derived gels. As shown in Figure $2 \mathrm{~d}$, larger amounts of plasma proteins are released from the gels at higher temperatures during the first 1-3 days of incubation. On the other hand, as shown in Figure $2 b$, fibrinogen contraction is highest at the lowest temperature. These contradictory effects could equalize the contraction presented by these gels at different temperatures. An alternative, or complementary, explanation could be that previously reported by Zhang et al. (2013) for collagen hydrogels, showing an outer hydrophilic and an inner hydrophobic part (Fang, Chen, Leu, \& Hu, 2008; Montero, Valencia, Corrales, Jorcano, \& Velasco, 2019). According to these authors, the aggregation of intermolecular assemblies when the hydrogels are submerged in an aqueous solution might be the mechanism behind contraction. In this environment, the nonpolar groups attract each other forming new crosslinking points that make the scaffold to be less porous and thus release more water to the environment, causing a mass decrease. However, contrary to what Zhang et al. report, we found that lower temperatures drove a more drastic contraction for our commercial fibrinogen model.

SEM analysis (Figure 3) also corroborate that hydrogel structure and behavior are greatly influenced by the presence/absence of embedded proteins. They also support the protein release kinetics data (Figures $2 \mathrm{~d}$ and S2). So, untreated plasma-based hydrogels (Figure 3a), containing its full complement of embedded proteins, present an overall matrix structure and a fiber structure and diameter (Figure S4) quite different from those of untreated commercial fibrinogen gels, lacking embedded proteins (Figure $3 d$ ). It seems that, under the SEM sample preparation conditions, the embedded proteins are adsorbed to, or precipitated around, the fibrin fibers. On the other hand, plasma-derived hydrogels treated for 7 days at $37^{\circ} \mathrm{C}$ (Figure 3c) and, therefore predicted to have lost most of their embedded proteins, look very similar to untreated commercial fibrinogen gels.

The rheological study performed at the three defined temperatures showed the absence of what is known as the strain hardening effect. This effect is defined as the increase of elastic modulus with strain amplitudes observed above $10 \%$ for fibrin hydrogels (Shah \& Janmey, 1997; Storm, Pastore, MacKintosh, Lubensky, \& Janmey, 2005). The molecular origin of the strain hardening seen in fibrin can be attributed to the rod-like or semiflexible nature of the fibrin strands in analogy to the polymer network theory of semiflexible chains (Litvinov \& Weisel, 2017). It is generally accepted that the onset of strain hardening relates to the straightening of these semiflexible filaments in the network upon straining and that this phenomenon is highly dependent on the structural organization of the fibrin hydrogel at the level of individual fibers (Li et al., 2016). The fact that strain-hardening behavior is not observed for plasma-derived hydrogels at any of the tested temperatures might again be attributed to the presence of proteins that alter the morphology and the number of crosslinking points responsible for the elastic response of the fibrin hydrogel as seen in the SEM images. This is an analogous result to that found for plasma clots as compared to pure fibrin hydrogels. In that case, the presence of platelets eliminated strain hardening in plasma clots (Shah \& Janmey, 1997).

All the samples present a constant value of $G^{\prime \prime}$ up to a value of strain (\%) where $G^{\prime \prime}$ starts to increase giving rise to a maximum, followed again by a decrease. This behavior is known as weak-strain hardening and it is directly related to the energy dissipation due to the breakage of a gel-like structure. In fibrin clot rheology, energy dissipation may arise from the dissociation of noncovalently linked adjacent fibrin monomers and the slippage of protofibrils or fibers between one another (Ryan, Mockros, Weisel, \& Lorand, 1999). The results found in Figure $4 \mathrm{~b}$ indicate that temperature increase leads to diminished levels of energy dissipation during deformation, that is, plasma-derived hydrogels become more rigid with temperature. These results might indicate that structural rearrangements between fibers 
or protofibrils become hindered with temperature and immersion time which could be related to a higher number of crosslinking points. In order to explain these results, it is necessary to take into account that lower temperatures favor fibrin polymerization thus leading to a lower protein release as demonstrated through protein release experiments (Figure 2d). However, the presence of proteins within plasma-derived fibrin hydrogels hinder the establishment of interactions between fibrin fibers resulting in higher times for gel-sol transition and lower deswelling rates with respect to fibrinogen hydrogels. Hence, the determinant factor for the observed rheological behavior seems to be the presence of proteins and the dependence of its release with temperature and time.

From the results obtained in Figure 4c,d, it can be inferred that the elastic properties of the prepared plasma-derived hydrogel remain unaltered when these are maintained at $4^{\circ} \mathrm{C}$, even after 7 days. This is possibly due to the increased fibrin polymerization at this temperature and the concomitant presence of proteins nonreleased with a reinforcing effect (see Figures $2 \mathrm{~d}$ and $3 \mathrm{~b}$ ). However, after 7 days, the plasma-derived fibrin gels present a lower elastic modulus due to the release of proteins that takes place when the gel is maintained at $T>23^{\circ} \mathrm{C}$, as demonstrated in the release profiles shown in Figure $2 \mathrm{~d}$, even if the formation of interactions between fibrin fibers is favored.

Finally, we wanted to understand the behavior of human-derived fibrin gels under conditions similar to those used in in vitro skin tissue culture. To this, we first studied gel contraction in DMEM culture medium supplemented with $10 \% \mathrm{FBS}$, at $37^{\circ} \mathrm{C}$. Under these conditions, plasma hydrogels contracted less than in PBS, as also reported in (Zhao et al., 2008).

Moreover, of the two deswelling phases repeatedly observed for these gels in PBS, only the early one, taking place typically during the initial 8-24 hr was detected. We attribute this change to the presence of calf serum proteins in the supplemented incubation medium, which would delay, prevent, or equilibrate the release of human plasma proteins from the fibrin matrix observed in PBS. Additionally, other differences in composition among DMEM and PBS could alter the hydrophylic-hydrophobic forces that, as also discussed earlier, could contribute to fibrin matrix contraction in PBS.

To approach in vitro skin cell culture conditions, plasma-derived hydrogels, more closely with and without skin cells, were prepared in transwell inserts as described in Figure 6c. Under these conditions, fibrin-derived hydrogels do not detach from the transwell walls nor from their bottom, contraction is manifested as a shrinking of the gel in the z-axis.

Interestingly, the z-axis hydrogel contraction during the initial $24 \mathrm{hr}$ was very similar regardless the presence of hFBS (Figure 6a). This effect was also similar to the early contraction displayed as mass loss by acellular, free-floating plasma-derived gels (Figures $2 \mathrm{a}$ and $5 \mathrm{a}$ ) and, therefore, contrary to what is widely accepted for the contraction of fibrin and collagen hydrogels, it cannot be attributed to fibroblasts, the only cells present in the gels at this early time. This result is also in agreement with the observation that hFBs no not have time enough to spread and attach to the fibrin matrix (Miron-Mendoza, Graham, Manohar, \& Petroll, 2017). At longer times, the effect of hFBs and
HKCs on the z-axis shrinkage becomes apparent, reaching very high values (up to $70 \%$ after 21 days) that limit the time span of the culture. We attribute this strong effect to cell-mediated contraction of the fibrin matrix together with matrix degradation by proteases synthesized by skin cells, such as matrix metalloproteinases. These enzymes are known to play important roles in proteolytic remodeling of ECM in various physiologic situations, including skin tissue repair and aging (Kähäri \& Saarialho-Kere, 1997; Pittayapruek, Meephansan, Prapapan, Komine, \& Ohtsuki, 2016).

\section{5 | CONCLUSIONS}

In this work, we have carefully analyzed the structure, rheological properties and behavior of plasma-derived fibrin hydrogels under different experimentally relevant conditions. The main aim was to delve into the causes behind the contraction that these gels experience over time, which is their main drawback and prevents a more widespread experimental and clinical use in wound healing and skin tissue engineering. Aware that the complex composition of human plasma would complicate these studies, we used commercial fibrinogen-based gels as controls.

The contractile behavior can be explained by the intrinsic tendency displayed by fibrin hydrogels to deswell in aqueous solution. This process is relatively quick and strongly dependent on temperature and can be very dramatic (e.g., 70\% mass loss after $8 \mathrm{hr}$ at $4^{\circ} \mathrm{C}$ ) for commercial fibrinogen-based fibrin gels. In the case of plasmaderived gels, the process is slowed down and milder (35\% contraction after 10 days), and, surprisingly, temperature-independent between 4 and $37^{\circ} \mathrm{C}$. We attribute this notorious change of behavior mainly to the progressive release to the incubation medium (PBS) of plasma proteins and lipids initially entrapped in the fibrin matrix, although remodeling of the fibrin matrix provoked by changes in hydrophilic/ hydrophobic interactions arising when the hydrogels are submerged in an aqueous solution could also be at play. In addition, this progressive depletion of plasma components from the fibrin gels seems to be involved in the changes that we observed in several parameters (e.g., pore size, fibrin fiber diameter, porosity, and rheological parameters) when incubating plasma-derived fibrin hydrogels at different temperatures for different times. These three mechanisms, namely, intrinsic fibrin matrix contraction, progressive depletion of plasma components, and changes in hydrophilic/hydrophobic forces, are also present in plasma-derived fibrin gels under conditions used for skin tissue culture. Together with additional matrix contraction and degradation due to the skin cells present in the culture, they lead to an excessive fibrin matrix reduction (70\% after 21 days in culture) that limits the usefulness of this skin tissue culture technique that clearly needs design improvements.

\section{ACKNOWLEDGMENTS}

The authors kindly thank Angélica del Corral and Sophia Lusia Marín for their technical assistance in the lab and Cristina Moral for her technical assistance with the SEM. This work was supported by 
Programa de Actividades de I + D entre Grupos de Investigación de la Comunidad de Madrid, S2018/BAA-4480, Biopieltec-CM, Programa Estatal de I + D + i Orientada a los Retos de la Sociedad, RTI2018101627-B-I00, Programa de Apoyo a la Realización de Proyectos Interdisciplinares de I+D para Jóvenes Investigadores de la Universidad Carlos III de Madrid (project: BIOMASKIN), and Cátedra Fundación Ramón Areces. The authors would like to acknowledge to the projects MAT2013-42957-R and MINECO (MAT201783014-C2-1-P).

\section{CONFLICT OF INTEREST}

The authors declare no potential conflict of interest.

\section{ORCID}

Diego Velasco (D) https://orcid.org/0000-0002-1531-1595

\section{REFERENCES}

Ahmed, T. A. E., Dare, E. V., \& Hincke, M. (2008). Fibrin: A versatile scaffold for tissue engineering applications. Tissue Engineering. Part $B$, Reviews, 14(2), 199-215.

Arnaud, P. (2004). The plasma proteins: Structure, function, and genetic control. Clinical Immunology and Immunopathology, 39(3), 531.

Auger, F. A., Lacroix, D., \& Germain, L. (2009). Skin substitutes and wound healing. Skin Pharmacology and Physiology, 22(2), 94-102.

Bell, E., Ivarsson, B., \& Merrill, C. (1979). Production of a tissue-like structure by contraction of collagen lattices by human fibroblasts of different proliferative potential in vitro. Proceedings of the National Academy of Sciences of the United States of America, 76(3), 1274-1278.

Bradford, M. M. (1976). A rapid and sensitive method for the quantitation of microgram quantities of protein utilizing the principle of proteindye binding. Analytical Biochemistry, 72(1-2), 248-254 Retrieved from https://www.sciencedirect.com/science/article/pii/ 0003269776905273

Braziulis, E., Diezi, M., Biedermann, T., Pontiggia, L., Schmucki, M., Hartmann-Fritsch, F., ... Reichmann, E. (2012). Modified plastic compression of collagen hydrogels provides an ideal matrix for clinically applicable skin substitutes. Tissue Engineering. Part C, Methods, 18(6), 464-474.

Brecher, M. E. (2005). Technical manual. 15th ed. Bethesda, MD: American Association of Blood Banks.

Buis, B., Koomen, G. C., Imholz, A. L., Struijk, D. G., Reddingius, R. E., Arisz, L., \& Krediet, R. T. (1996). Effect of electric charge on the transperitoneal transport of plasma proteins during CAPD. Nephrology, Dialysis, Transplantation, 11(6), 1113-1120.

Burmeister, D. M., Roy, D. C., Becerra, S. C., Natesan, S., \& Christy, R. J. (2018). In situ delivery of fibrin-based hydrogels prevents contraction and reduces inflammation. Journal of Burn Care \& Research, 39(1) 40-53.

Burmeister, D. M., Stone, R., Wrice, N. L., Becerra, S. C., Natesan, S., \& Christy, R. J. (2016). Fibrin hydrogels prevent contraction and deliver adipose stem cells to debrided deep partial thickness burns for accelerated angiogenesis. FASEB Journal, 30(Suppl 1), 1300-1307.

Campbell, P. K., Bennett, S. L., Driscoll, A., \& Sawhney, A. S. (2005). Evaluation of absorbable surgical sealants: In-vitro testing, (pp. 1-4). Whaltam, MA: Confluent Surgical Inc.

Caravaggi, C., De Giglio, R., Pritelli, C., Sommaria, M., Dalla Noce, S., Faglia, E., Morabito, A. (2003). HYAFF 11-Based Autologous Dermal and Epidermal Grafts in the Treatment of Noninfected Diabetic Plantar and Dorsal Foot Ulcers: A prospective, multicenter, controlled, randomized clinical trial. Diabetes Care, 26(10), 2853-2859. http://dx.doi. org/10.2337/diacare.26.10.2853.
Carriel, V., Garzón, I., Jiménez, J.-M., Oliveira, C.-X., Arias-Santiago, S., Campos, A., Alaminos, M. (2011). Epithelial and Stromal Developmental Patterns in a Novel Substitute of the Human Skin Generated with Fibrin-Agarose Biomaterials. Cells Tissues Organs, 196(1), 1-12. http:// dx.doi.org/10.1159/000330682.

Cen, L., Liu, W. E. I., Cui, L. E. I., Zhang, W., \& Cao, Y. (2008). Collagen tissue engineering: Development of novel biomaterials and applications. Pediatric Research, 63(5), 492-496.

Centanni, J. M., Straseski, J. A., Wicks, A., Hank, J. A., Rasmussen, C. A., Lokuta, M. A., ... Allen-Hoffmann, B. L. (2011). StrataGraft skin substitute is well-tolerated and is not acutely immunogenic in patients with traumatic wounds: Results from a prospective, randomized, controlled dose escalation trial. Annals of Surgery, 253(4), 672-683.

Chaudhari, A. A., Vig, K., Baganizi, D. R., Sahu, R., Dixit, S., Dennis, V., \& Shreekumar, R. P. (2016). Future prospects for scaffolding methods and biomaterials in skin tissue engineering: A Review. International Journal of Molecular Sciences, 17(12), 1974

Chen, Y., Shi-Wen, X., van Beek, J., Kennedy, L., McLeod, M., Renzoni, E. A., \& Leask, A. (2005). Matrix contraction by dermal fibroblasts requires transforming growth factor-beta/activin-linked kinase 5, heparan sulfate-containing proteoglycans, and MEK/ERK: Insights into pathological scarring in chronic fibrotic disease. The American Journal of Pathology, 167(6), 1699-1711.

Chua, A. W. C., Khoo, Y. C., Tan, B. K., Tan, K. C., Foo, C. L., \& Chong, S. J. (2016). Skin tissue engineering advances in severe burns: Review and therapeutic applications. Burn Trauma, 4, 3.

Cohen, A. L. (1979). Critical point drying-principles and procedures. Scanning Electron Microscopy, 2, 303-324.

Cubo, N., Garcia, M., del Canizo, J. F., Velasco, D., \& Jorcano, J. L. (2016). 3D bioprinting of functional human skin: Production and in vivo analysis. Biofabrication, 9(1), 15006.

Curran, M. P., \& Plosker, G. L. (2002). Bilayered bioengineered skin substitute (Apligraf): A review of its use in the treatment of venous leg ulcers and diabetic foot ulcers. BioDrugs, 16(6), 439-455.

Darby, I. A., Zakuan, N., Billet, F., \& Desmouliere, A. (2016). The myofibroblast, a key cell in normal and pathological tissue repair. Cellular and Molecular Life Sciences, 73(6), 1145-1157.

de Jesus, A. M., \& Sander, E. A. (2014). Observing and quantifying fibroblast-mediated fibrin gel compaction. Journal of Visualized Experiments, 83, e50918.

del Rio, M., Larcher, F., Serrano, F., Meana, A., Muñoz, M., Garcia, M., \& Jorcano, J. L. (2002). A preclinical model for the analysis of genetically modified human skin in vivo. Human Gene Therapy, 13(8), 959-968.

Fang, J.-Y., Chen, J.-P., Leu, Y.-L., \& Hu, J.-W. (2008). Temperature-sensitive hydrogels composed of chitosan and hyaluronic acid as injectable carriers for drug delivery. European Journal of Pharmaceutics and Biopharmaceutics, 68(3), 626-636.

Ferrer-Mayorga, G., Gomez-Lopez, G., Barbachano, A., FernandezBarral, A., Pena, C., Pisano, D. G., \& Larriba, M. J. (2017). Vitamin D receptor expression and associated gene signature in tumour stromal fibroblasts predict clinical outcome in colorectal cancer. Gut, 66(8), 1449-1462.

Fischer, E. G., Ruf, W., \& Mueller, B. M. (1995). Tissue factor-initiated thrombin generation activates the signaling thrombin receptor on malignant melanoma cells. Cancer Research, 55(8), 1629-1632.

Freyman, T. M., Yannas, I. V., Yokoo, R., \& Gibson, L. J. (2001). Fibroblast contraction of a collagen-GAG matrix. Biomaterials, 22(21), 2883-2891.

Gaffney, P. J., \& Edgell, T. A. (1995). The international and "NIH" units for thrombin-How do they compare? Thrombosis and Haemostasis, 74(3), 900-903.

Glowacki, J., \& Mizuno, S. (2008). Collagen scaffolds for tissue engineering. Biopolymers, 89(5), 338-344.

Grinnell, F. (2000). Fibroblast-collagen-matrix contraction: Growth-factor signalling and mechanical loading. Trends in Cell Biology, 10 (9), 362 
Groeber, F., Holeiter, M., Hampel, M., Hinderer, S., \& Schenke-Layland, K. (2011). Skin tissue engineering-In vivo and in vitro applications. Advanced Drug Delivery Reviews, 63(4-5), 352-366.

Guerrero-Aspizua, S., Garcia, M., Murillas, R., Retamosa, L., Illera, N., Duarte, B., \& del Río, M. (2010). Development of a bioengineered skin-humanized mouse model for psoriasis: Dissecting epidermal-lymphocyte interacting pathways. The American Journal of Pathology, 177 (6), 3112-3124.

Haisch, A., Klaring, S., Groger, A., Gebert, C., \& Sittinger, M. (2002). A tissue-engineering model for the manufacture of auricular-shaped cartilage implants. European Archives of Oto-Rhino-Laryngology, 259(6), 316-321.

Haugh, M. G., Thorpe, S. D., Vinardell, T., Buckley, C. T., \& Kelly, D. J. (2012). The application of plastic compression to modulate fibrin hydrogel mechanical properties. Journal of the Mechanical Behavior of Biomedical Materials, 16, 66-72.

Insenser, M., Martinez-Garcia, M. A., Nieto, R. M., San-Millan, J. L., \& Escobar-Morreale, H. F. (2010). Impact of the storage temperature on human plasma proteomic analysis: Implications for the use of human plasma collections in research. Proteomics. Clinical Applications, 4(8-9), 739-744.

Jansen, K. A., Bacabac, R. G., Piechocka, I. K., \& Koenderink, G. H. (2013). Cells actively stiffen fibrin networks by generating contractile stress. Biophysical Journal, 105(10), 2240-2251.

Javelaud, D., Laboureau, J., Gabison, E., Verrecchia, F., \& Mauviel, A. (2003). Disruption of basal JNK activity differentially affects key fibroblast functions important for wound healing. The Journal of Biological Chemistry, 278(27), 24624-24628.

Jeong, K.-H., Park, D., \& Lee, Y.-C. (2017). Polymer-based hydrogel scaffolds for skin tissue engineering applications: A mini-review. Journal of Polymer Research, 24(7), 112.

Kähäri, V., \& Saarialho-Kere, U. (1997). Matrix metalloproteinases in skin. Experimental Dermatology, 6(5), 199-213.

Kopp, J., Preis, E., Said, H., Hafemann, B., Wickert, L., Gressner, A. M., ... Dooley, S. (2005). Abrogation of transforming growth factor-beta signaling by SMAD7 inhibits collagen gel contraction of human dermal fibroblasts. The Journal of Biological Chemistry, 280(22), 2157021576.

Kreger, S. T., Bell, B. J., Bailey, J., Stites, E., Kuske, J., Waisner, B., \& Voytik-Harbin, S. L. (2010). Polymerization and matrix physical properties as important design considerations for soluble collagen formulations. Biopolymers, 93(8), 690-707.

Li, B., \& Wang, J. H.-C. (2011). Fibroblasts and myofibroblasts in wound healing: Force generation and measurement. Journal of Tissue Viability, 20(4), 108-120.

Li, W., Sigley, J., Pieters, M., Helms, C. C., Nagaswami, C., Weisel, J. W., \& Guthold, M. (2016). Fibrin fiber stiffness is strongly affected by fiber diameter, but not by fibrinogen glycation. Biophysical Journal, 110(6), 1400-1410.

Litvinov, R. I., \& Weisel, J. W. (2017). Fibrin mechanical properties and their structural origins. Matrix Biology, 60-61, 110-123.

Llames, S., Garcia, E., Garcia, V., del Rio, M., Larcher, F., Jorcano, J. L., \& Meana, A. (2006). Clinical results of an autologous engineered skin. Cell and Tissue Banking, 7(1), 47-53.

Llames, S. G., del Rio, M., Larcher, F., Garcia, E., Garcia, M., Escamez, M. J., \& Meana, A. (2004). Human plasma as a dermal scaffold for the generation of a completely autologous bioengineered skin. Transplantation, 77(3), 350-355.

Lotz, C., Schmid, F. F., Oechsle, E., Monaghan, M. G., Walles, H., \& Groeber-Becker, F. (2017). Cross-linked collagen hydrogel matrix resisting contraction to facilitate full-thickness skin equivalents. ACS Applied Materials \& Interfaces, 9(24), 20417-20425.

MacNeil, S. (2007). Progress and opportunities for tissue-engineered skin. Nature, 445(7130), 874-880.
MacNeil, S. (2008). Biomaterials for tissue engineering of skin. Materials Today, 11(5), 26-35 Retrieved from: https://www.sciencedirect.com/ science/article/pii/S1369702108700877

Mandrycky, C., Wang, Z., Kim, K., \& Kim, D.-H. (2016). 3D bioprinting for engineering complex tissues. Biotechnology Advances, 34(4), 422-434.

Martinez-Santamaria, L., Conti, C. J., Llames, S., Garcia, E., Retamosa, L., Holguin, A., \& del Río, M. (2013). The regenerative potential of fibroblasts in a new diabetes-induced delayed humanised wound healing model. Experimental Dermatology, 22(3), 195-201.

Mathes, S. H., Ruffner, H., \& Graf-Hausner, U. (2014). The use of skin models in drug development. Advanced Drug Delivery Reviews, 69-70, 81-102.

Meana, A., Iglesias, J., del Rio, M., Larcher, F., Madrigal, B., Fresno, M. F., \& Tevar, F. (1998). Large surface of cultured human epithelium obtained on a dermal matrix based on live fibroblast-containing fibrin gels. Burns, 24(7), 621-630.

Metcalfe, A. D., \& Ferguson, M. W. J. (2007). Tissue engineering of replacement skin: The crossroads of biomaterials, wound healing, embryonic development, stem cells and regeneration. Journal of the Royal Society Interface, 4(14), 413-437.

Miron-Mendoza, M., Graham, E., Manohar, S., \& Petroll, W. M. (2017). Fibroblast-fibronectin patterning and network formation in 3D fibrin matrices. Matrix Biology, 64, 69-80.

Monaghan, M., Browne, S., Schenke-Layland, K., \& Pandit, A. (2014). A collagen-based scaffold delivering exogenous microrna-29B to modulate extracellular matrix remodeling. Molecular Therapy, 22(4), 786-796.

Montero, A., Valencia, L., Corrales, R., Jorcano, J. L., \& Velasco, D. (2019). Smart polymer gels: Properties, synthesis, and applications. In Smart polymers and their applications (pp. 279-321). Sawston, Cambridge: Woodhead Publishing in Materials. Retrieved from https://www. sciencedirect.com/science/article/pii/B9780081024164000090

Mori, N., Morimoto, Y., \& Takeuchi, S. (2017). Skin integrated with perfusable vascular channels on a chip. Biomaterials, 116, 48-56.

Natesan, S., Stone, R., Coronado, R. E., Wrice, N. L., Kowalczewski, A. C., Zamora, D. O., \& Christy, R. J. (2019). PEGylated platelet-free blood plasma-based hydrogels for full-thickness wound regeneration. Advances in Wound Care, 8(7), 323-340. https://doi.org/10.1089/ wound.2018.0844

Niederman, R., Amrein, P. C., \& Hartwig, J. (1983). Three-dimensional structure of actin filaments and of an actin gel made with actin-binding protein. The Journal of Cell Biology, 96(5), 1400-1413.

Norouzi, M., Boroujeni, S. M., Omidvarkordshouli, N., \& Soleimani, M. (2015). Advances in skin regeneration: Application of electrospun scaffolds. Advanced Healthcare Materials, 4(8), 1114-1133.

Pinto, J., Domingues, M. R. M., Galhano, E., Pita, C., Almeida, M., Carreira, I. M., \& Gil, A. M. (2014). Human plasma stability during handling and storage: Impact on NMR metabolomics. The Analyst, 139(5), 1168-1177.

Pittayapruek, P., Meephansan, J., Prapapan, O., Komine, M., \& Ohtsuki, M. (2016). Role of matrix metalloproteinases in photoaging and photocarcinogenesis. International Journal of Molecular Sciences, 17(6), 868.

Priya, S. G., Jungvid, H., \& Kumar, A. (2008). Skin tissue engineering for tissue repair and regeneration. Tissue Engineering. Part B, Reviews, 14(1), 105-118.

Rheinwatd, J. G., \& Green, H. (1975). Seria cultivation of strains of human epidemal keratinocytes: The formation keratinizin colonies from single cell is. Cell, 6(3), 331-343.

Ruszymah, B. H. I., Chua, K. H., Mazlyzam, A. L., \& Aminuddin, B. S. (2011). Formation of tissue engineered composite construct of cartilage and skin using high density polyethylene as inner scaffold in the shape of human helix. International Journal of Pediatric Otorhinolaryngology, 75(6), 805-810.

Ryan, E. A., Mockros, L. F., Weisel, J. W., \& Lorand, L. (1999). Structural origins of fibrin clot rheology. Biophysical Journal, 77(5), 2813-2826. 
Shah, J. V., \& Janmey, P. A. (1997). Strain hardening of fibrin gels and plasma clots. Rheologica Acta, 36(3), 262-268.

Shevchenko, R. V., James, S. L., \& James, S. E. (2010). A review of tissueengineered skin bioconstructs available for skin reconstruction. Journal of the Royal Society Interface, 7(43), 229-258.

Shores, J. T., Gabriel, A., \& Gupta, S. (2007). Skin substitutes and alternatives: A review. Advances in Skin \& Wound Care, 20(9 Pt 1), 410-493.

Sriram, G., Alberti, M., Dancik, Y., Wu, B., Wu, R., Feng, Z., ... Wang, Z. (2018). Full-thickness human skin-on-chip with enhanced epidermal morphogenesis and barrier function. Materials Today, 21(4), 326-340.

Stark, H.-J., Boehnke, K., Mirancea, N., Willhauck, M. J., Pavesio, A., Fusenig, N. E., \& Boukamp, P. (2006). Epidermal homeostasis in longterm scaffold-enforced skin equivalents. Journal of Investigative Dermatology Symposium Proceedings, 11(1), 93-105.

Still, J., Glat, P., Silverstein, P., Griswold, J., \& Mozingo, D. (2003). The use of a collagen sponge/living cell composite material to treat donor sites in burn patients. Burns, 29(8), 837-841.

Stojic, M., López, V., Montero, A., Quílez, C., de Aranda, I. G., Vojtova, L., \& Velasco, D. (2019). Skin tissue engineering. Biomaterials for Skin Repair and Regeneration, (pp. 59-99). Sawston, Cambridge: Woodhead Publishing. Retrieved from: https://www.sciencedirect.com/science/ article/pii/B9780081025468000030

Stone, R., Wall, J., Natesan, S., \& Christy, R. (2018). PEG-plasma hydrogels increase epithelialization using a human ex vivo skin model. International Journal of Molecular Sciences, 19(10), 3156.

Storm, C., Pastore, J. J., MacKintosh, F. C., Lubensky, T. C., \& Janmey, P. A (2005). Nonlinear elasticity in biological gels. Nature, 435(7039), 191-194.

Student. (1908). The probable error of a mean. Biometrika, 6(1), 1-25.

Supp, D. M., \& Boyce, S. T. (2005). Engineered skin substitutes: Practices and potentials. Clinics in Dermatology, 23(4), 403-412.

Syverud, K., Kirsebom, H., Hajizadeh, S., \& Chinga-Carrasco, G. (2011). Cross-linking cellulose nanofibrils for potential elastic cryo-structured gels. Nanoscale Research Letters, 6(1), 626.

Tracy, L. E., Minasian, R. A., \& Caterson, E. J. (2016). Extracellular matrix and dermal fibroblast function in the healing wound. Advances in Wound Care, 5(3), 119-136.

Tuan, T.-L., Song, A., Chang, S., Younai, S., \& Nimni, M. E. (1996). In vitrofibroplasia: Matrix contraction, cell growth, and collagen production of fibroblasts cultured in fibrin gels. Experimental Cell Research, 223(1), 127-134.

Turner, P., McLennan, A., Bates, A., \& White, M. (2007). BIOS instant notes in molecular biology. BIOS instant notes in molecular biology, 3, (360). London, UK: Taylor \& Francis.

Velasco, D., Quílez, C., Garcia, M., del Cañizo, J. F., \& Jorcano, J. L. (2018). 3D human skin bioprinting: A view from the bio side. Journal of 3D Printing in Medicine, 2(3), 141-162. https://doi.org/10.2217/3dp2018-0008

Vig, K., Chaudhari, A., Tripathi, S., Dixit, S., Sahu, R., Pillai, S., \& Shree, R. S. (2017). Advances in skin regeneration using tissue engineering. International Journal of Molecular Sciences, 18(4), 789
Walker, R. H. (1993). Technical manual. Bethesda, MD: American Association of Blood Banks. Department of the Army Technical Manual; TM 8-227-3.

Wedgwood, J., Freemont, A. J., \& Tirelli, N. (2013). Rheological and turbidity study of fibrin hydrogels. Macromolecular Symposia, 334(1), 117125. https://doi.org/10.1002/masy.201300111

Weigandt, K. M., White, N., Chung, D., Ellingson, E., Wang, Y., Fu, X., \& Pozzo, D. C. (2012). Fibrin clot structure and mechanics associated with specific oxidation of methionine residues in fibrinogen. Biophysical Journal, 103(11), 2399-2407 Retrieved from https://www.ncbi. nlm.nih.gov/pubmed/23283239

Wynn, T. A., \& Ramalingam, T. R. (2012). Mechanisms of fibrosis: Therapeutic translation for fibrotic disease. Nature Medicine, 18(7), 1028-1040.

Yang, T.-H., Thoreson, A. R., Gingery, A., An, K.-N., Larson, D. R., Zhao, C., \& Amadio, P. C. (2015). Collagen gel contraction as a measure of fibroblast function in carpal tunnel syndrome. Journal of Biomedical Materials Research. Part A, 103(2), 574-580.

Yu, J. R., Navarro, J., Coburn, J. C., Mahadik, B., Molnar, J., Holmes, J. H., IV, \& Fisher, J. P. (2019). Current and future perspectives on skin tissue engineering: Key features of biomedical research, translational assessment, and clinical application. Advanced Healthcare Materials, 8 (5), 1801471. https://doi.org/10.1002/adhm.201801471

Zhang, X., Xu, L., Wei, S., Zhai, M., \& Li, J. (2013). Stimuli responsive deswelling of radiation synthesized collagen hydrogel in simulated physiological environment. Journal of Biomedical Materials Research. Part A, 101(8), 2191-2201.

Zhao, H., Ma, L., Zhou, J., Mao, Z., Gao, C., \& Shen, J. (2008). Fabrication and physical and biological properties of fibrin gel derived from human plasma. Biomedical Materials, 3(1), 15001.

Zhu, Y. K., Umino, T., Liu, X. D., Wang, H. J., Romberger, D. J., Spurzem, J. R., \& Rennard, S. I. (2001). Contraction of fibroblast-containing collagen gels: Initial collagen concentration regulates the degree of contraction and cell survival. In Vitro Cellular \& Developmental Biology. Animal, 37(1), 10-16.

Zor, T., \& Selinger, Z. (1996). Linearization of the Bradford protein assay increases its sensitivity: Theoretical and experimental studies. Analytical Biochemistry, 236(2), 302-308.

\section{SUPPORTING INFORMATION}

Additional supporting information may be found online in the Supporting Information section at the end of this article.

How to cite this article: Montero A, Acosta S, Hernández R, Elvira C, Jorcano JL, Velasco D. Contraction of fibrin-derived matrices and its implications for in vitro human skin bioengineering. J Biomed Mater Res. 2021;109A:500-514. https://doi.org/10.1002/jbm.a.37033 